\title{
The Remarkable Structural Diversity Achieved in ent-Kaurane Diterpenes by Fungal Biotransformations
}

\author{
Jacqueline A. Takahashi ${ }^{1, *}$, Dhionne C. Gomes ${ }^{2}$, Fernanda H. Lyra ${ }^{1}$, Gabriel F. dos Santos ${ }^{1}$ \\ and Leonardo R. Martins ${ }^{3}$
}

1 Departamento de Química, Universidade Federal de Minas Gerais (UFMG), Av. Antonio Carlos, 6627, CEP 31270-901, Belo Horizonte, M.G., Brazil; E-Mails: fernandahl@yahoo.com.br (F.H.L.); gfsantos@ymail.com (G.F.S.)

2 Faculdade de Farmácia, Universidade Federal de Minas Gerais (UFMG), Av. Antonio Carlos, 6627, CEP 31270-901, Belo Horizonte, M.G., Brazil; E-Mail: dhionnegomes@hotmail.com

3 Faculdade de Ciências Exatas e Tecnologia (FACET), Universidade Federal da Grande Dourados (UFGD), Rodovia Dourados-Itahum, km 12, CEP 79.804-970, Cx. Postal 533, Dourados, M.S., Brazil; E-Mail: leonardomartins@ufgd.edu.br

* Author to whom correspondence should be addressed; E-Mail: jat@qui.ufmg.br; Tel.: +5531-3409-5754; Fax: +5531-3409-5700.

Received: 24 December 2013; in revised form: 5 February 2014 / Accepted: 6 February 2014 / Published: 10 February 2014

\begin{abstract}
The use of biotransformations in organic chemistry is widespread, with highlights of interesting applications in the functionalization of natural products containing unactivated carbons, like the kaurane diterpenes. A number of compounds with kaurane skeletons can be isolated in large amounts from several plant species and a myriad of biological activities has been related to these compounds. Studies on structure versus activity have showed that, in most cases, in kaurane diterpenes, activity increases with the increase of functionalization. Since naturally occurring kaurane diterpenes usually have limited functional groups to be used as targets for semi-synthetic modifications, production of more polar derivatives from kaurane diterpenes have been achieved mostly through the use of fungal biotransformations. In this review, selected examples the wonderful chemical diversity produced by fungi in kaurane diterpenes is presented. This diversity includes mainly hydroxylation of nearly all carbon atoms of the kaurane molecule, many of them carried out stereoselectively, as well as ring rearrangements, among other chemical modifications. Sources of starting materials, general biotransformation protocols employed,
\end{abstract}


fungi with most consistent regioselectivity towards kaurane skeleton, as well as biological activities associated with starting materials and products are also described.

Keywords: biotransformation; kaurane diterpenes; filamentous fungi; hydroxylation; structure diversification

\section{Introduction}

One of the major aims of natural products chemistry is the isolation of molecules with biological properties. With the development of novel strategies for natural products research, especially biomonitored isolation and the use of preparative/hyphenated High Performance Liquid Chromatography (HPLC) there was a speed up in the discovery process, leading to a number of new molecules of diversified structures displaying an extensive range of bioactivities. At the same time, organic synthesis is producing novel molecules throughout total synthesis as well as a number of natural product derivatives for structure-activity studies. Biotransformations have an interesting role in a gap left between these two approaches, since they enable chemical modifications, mainly the introduction of hydroxyl groups, at inactivated carbons, which is a difficult task for semi-synthesis. The biotransformation approach has vast application in terpene molecules, a group of natural compounds possessing a myriad of biological activities, but however bearing only a few reactive sites for chemical modifications. Low polarity terpenes are usually available from natural sources in large amounts, making possible their prompt use in biotransformations. Biotransformation represents the second generation process of choice in the manufacture of small molecules with pharmaceutical applications [1].

The biotransformation of terpenes by fungi has applications in the most diverse areas, such as the pharmaceutical, food, cosmetics, and fragrance industries [2,3]. Nearly all classes of terpenes have been successfully functionalized by biotransformations. For instance, a monoterpene commonly targeted is limonene, due to its wide availability from cheap sources such as waste from the orange juice industry and its great potential as a substrate for the production of fragrances [4]. Functionalized derivatives of sesquiterpenes [5,6] and triterpenes [7] have also been obtained as fungal metabolites. Reports on the biotransformation of many different types of diterpenes, such as labdane [8], trachylobane [9,10], stemodane [11], aphidicolane [12], pimaradiene [13], abietane [14], taxane [15], atisane [16], and beyerane [17] are also very frequently found in the literature.

Kaurane diterpenes represent a target of interest for biotransformations because they constitute a class of substances rich in different biological activities [18]; some of them also act as intermediates in the biosynthesis of some fungal metabolites. The use of kaurane diterpenes in microbiological conversions is mainly directed to biosynthesis studies and to the production of derivatives with biological activities. In Table 1, an overview of some key biological activities related to kaurane diterpenes is presented. 
Table 1. Biological activities associated with kaurane diterpenes.

\begin{tabular}{|c|c|c|c|c|}
\hline Compound & Type & $\begin{array}{l}\text { Plant or fungi } \\
\text { (biotransformation) source }\end{array}$ & Biological activities & Reference \\
\hline ent-18-acetoxykaur-16-ene & NP & Annona squamosa & anti-inflammatory and analgesic & [19] \\
\hline 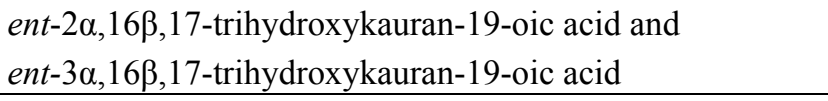 & NP & Mikania hirsutissima & immunomodulatory (human lymphocytes) & {$[20]$} \\
\hline ent-3 $3,15 \beta, 18$-trihydroxykaur-16-ene & $\mathrm{BP}$ & Mucor plumbeus (fungus) & anti-allergic & {$[21]$} \\
\hline $\begin{array}{l}\text { ent } 7 \beta, 16 \beta, 17 \text {-trihydroxykauran-6-one and } \\
\text { ent-7 } \alpha, 16 \beta, 17 \text {-trihydroxykauran-6-one }\end{array}$ & NP & Broussonetia papyrifera & anti-tyrosinase & {$[22]$} \\
\hline ent-13,16 $\beta, 17$-trihydroxykauran-19-oic acid & $\mathrm{BP}$ & Mucor recurvatus (fungus) & glucocorticoid agonists & {$[23]$} \\
\hline 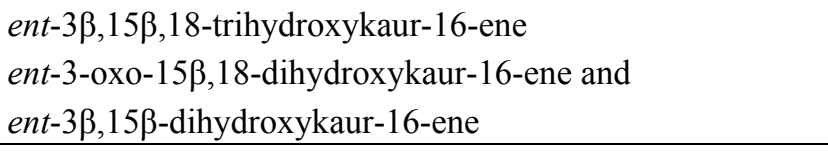 & NP & Suregada multiflora & anti-allergic & {$[24]$} \\
\hline 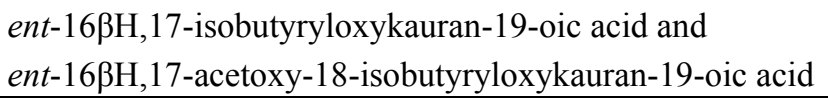 & NP & Siegesbeckia glabrescens & antidiabetic and antiobesity & {$[25]$} \\
\hline ent-2 $\alpha$-hydroxy,16-oxo-17-norkauran-19-oic acid & $\mathrm{BP}$ & Fusarium proliferatum (fungus) & allelopathic & {$[26]$} \\
\hline $\begin{array}{l}\text { ent-7 } \alpha, 11 \beta \text {-dihydroxykaur-16-en-19-oic acid and } \\
\text { ent-1 } \beta, 7 \alpha \text {-dihydroxykaur-16-en-19-oic acid }\end{array}$ & $\mathrm{BP}$ & Aspergillus niger (fungus) & spasmolytic & {$[27]$} \\
\hline 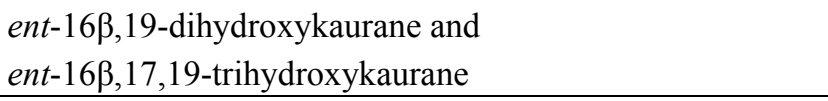 & $\mathrm{BP}$ & Cephalosporium aphidicola (fungus) & allelopathic & {$[28]$} \\
\hline \multirow{8}{*}{ ent-kaur-16-en-19-oic acid (kaurenoic acid) } & NP & Aspilia foliacea & antimicrobial & [29] \\
\hline & NP & $\begin{array}{l}\text { Mikania obtusata, Xylopia frutescens, } \\
\text { X. sericea and Wedelia paludosa }\end{array}$ & trypanocidal & {$[30,31]$} \\
\hline & NP & Melantheria albinervia & larvicidal & [32] \\
\hline & NP & Annona glabra & $\begin{array}{l}\text { antimicrobial, antifungal, } \\
\text { antihelmintic and sporicidal }\end{array}$ & {$[33]$} \\
\hline & NP & $W \cdot$ paludosa & antinociceptive & [34] \\
\hline & NP & Copaifera langsdorffii & cytotoxic and genotoxic & {$[35,36]$} \\
\hline & NP & W. paludosa & anti-inflammatory & {$[37]$} \\
\hline & NP & Laetia thamnia & anti-Parkinsonism & {$[38]$} \\
\hline
\end{tabular}

NP: Natural Product isolated from plants; BP: Biotransformation Product obtained using fungi. 
Among these compounds, ent-kaur-16-en-19-oic acid (kaurenoic acid, 1, Figure 1) is one of the most studied compounds, as it has several and varied biological activities, such as antimicrobial [29], inhibition of HIV replication [39], anti-inflammatory [40], trypanocidal [41] and cytotoxic properties [42], among others.

Figure 1. Chemical structure of kaurenoic acid.

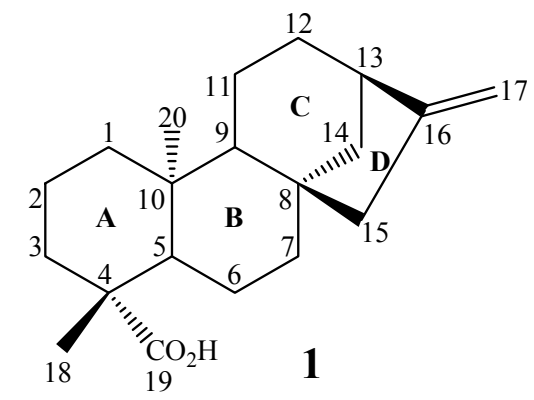

Other kaurane diterpenes like steviol (2), steviol 16 $\alpha$,17-epoxide (3) and stevioside (4) (Figure 2), showed anti-inflammatory activity when screened as glucocorticoid agonists [43].

Figure 2. Chemical structures of steviol (2), steviol 16 $\alpha$, 17-epoxide (3) and stevioside (4).

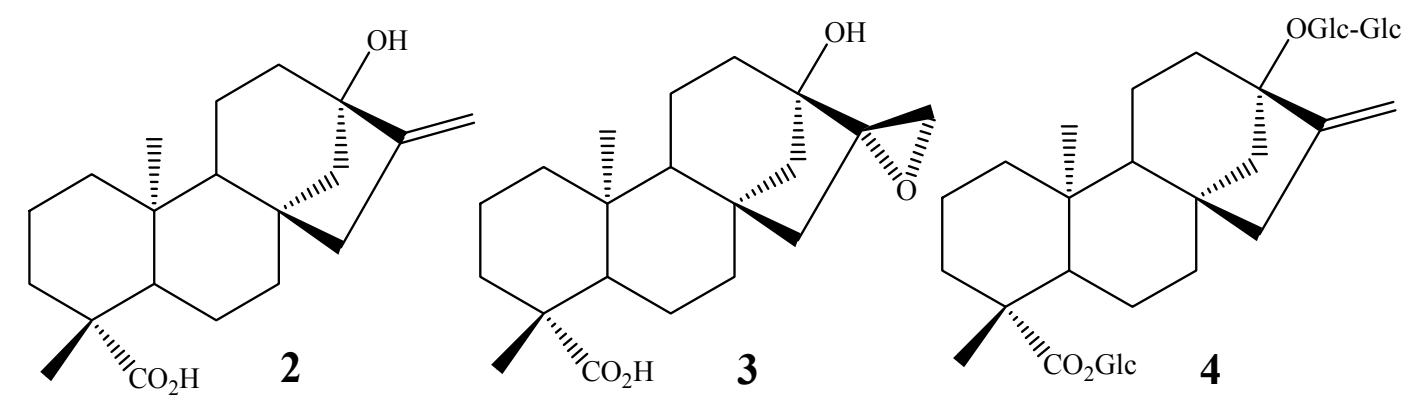

Hydroxylated kaurane diterpenes are of special interest, as oxygenated derivatives generally have higher levels of biological activity than their non-polar precursors. For instance, Ohkoshi et al. reported significant immunomodulatory activity towards human lymphocytes for kaurenoic acid derivatives hydroxylated in carbons 2, 3, 16 and 17 [20]. Kaurane compounds bearing a $6 \beta-\mathrm{OH}$ group that establishes a hydrogen bond with a C-15 carbonyl group have been associated to antitumor activity as well as antibacterial activity against Gram-positive bacteria [44].

Evaluation of the relationship between structure and antimicrobial activity of some diterpenes suggested that the presence of a substituted decalin skeleton (hydrophobic portion), and a hydrophilic region bearing a donor-group able to interact with hydrogen-bond acceptor groups on the membrane were necessary for their insertion in a phospholipid bilayer model. This feature enables the diterpenes to cross the bacterial cell membrane causing cell damage [45].

Other studies reported that increase in the antitumor activity in kaurane diterpenes is related to the presence of hydroxyl groups at C-7 and/or C-14 [46,47]. Polyhydroxylated kaurane diterpenes like oridonin (5, Figure 3) present anti-tumor activity against P 388 lymphocytic leukemia in mice, and increases in the degree of functionalization have been associated with higher antitumor activity [48]. 
Figure 3. Chemical structures of compounds 5 and 6.

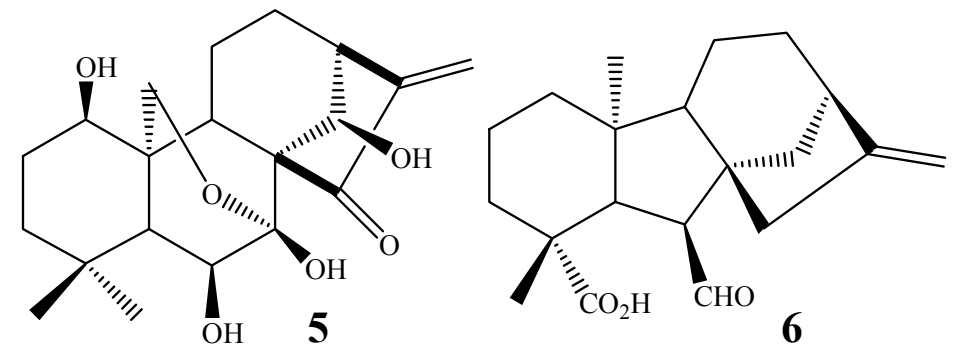

The hydroxylation of $\mathrm{C}-11$ in kaurane diterpenes is frequently accomplished by fungal biotransformation. These molecules hold some similarities to the steroidal nucleus. The presence of a hydroxyl group at C-11 in the steroidal nucleus is a structural requirement for carbohydrate-regulating hormonal activity of the adrenal steroids [49]. Cortisol and aldosterone are good examples of steroidal hormones hydroxylated at C-11, and hydroxylation of these molecules is catalyzed by $11 \beta$-hydroxylase and aldosterone synthase enzymes that belong to the cytochrome P 450 complex [50].

\section{Biotransformations of Kaurane Diterpenes by Fungi from Gibberella fujikuroi Complex}

Many biotransformations of kaurane diterpenes described in the literature have been carried out by G. fujikuroi. The advancement of genetic sequencing techniques allowed gathering at least 50 different species of fungi of Fusarium genus including $F$. proliferatum, F. acutatum and F. fujikuroi within a group called G. fujikuroi complex [51]. This group of fungi has the enzymatic system conducive to carry on the same type of biotransformation, and is reported as producers of diterpenes [52]. The G. fujikuroi complex was gathered due to the need to reclassify different species of fungi from Fusarium genus, which were classified according to the same taxonomy, as they possessed identical morphological features.

The kaurane diterpenes are produced through the mevalonate pathway. The first metabolite of this via contains a tetracyclic skeleton, produced from geranylgeranyl pyrophosphate through the action of the enzyme kaurene synthetase. Post-kaurene modifications are related to the cytochrome P-450, for example, oxygenases [52] and monooxygenases [53]. Kaurane diterpenes, like kaurenoic acid, are natural intermediates in the in vivo biosynthesis of gibberellins. Therefore, some kaurane diterpenes, under the action of fungi from G. fujikuroi complex, are hydroxylated in C-7, following a rearrangement with ring $\mathrm{B}$ contraction, into a five-membered ring, due to extrusion of $\mathrm{C}-7$, providing gibberellins [54]. The gibberellins are tetracyclic diterpenes present in low concentrations in plants, where they regulate different stages of growth and development. An example is the production of ent7-oxo-gibb-16-ene (6) (Figure 3) by the biotransformation of a kaurane precursor by G. fujikuroi [55]. The production of gibberellins from the biotransformation of kaurane diterpenes is of great importance and high commercial value, mainly because gibberellins natural production occurs in very small amounts [56,57].

Fungi from G. fujikuroi complex were used as biocatalysts not only for producing gibberellins, but also for the hydroxylation of different positions, as shown in Figure 4. 
Figure 4. Positions and orientations of hydroxyl groups added to kaurane diterpenes by biotransformations using fungi from G. fujikuroi complex.

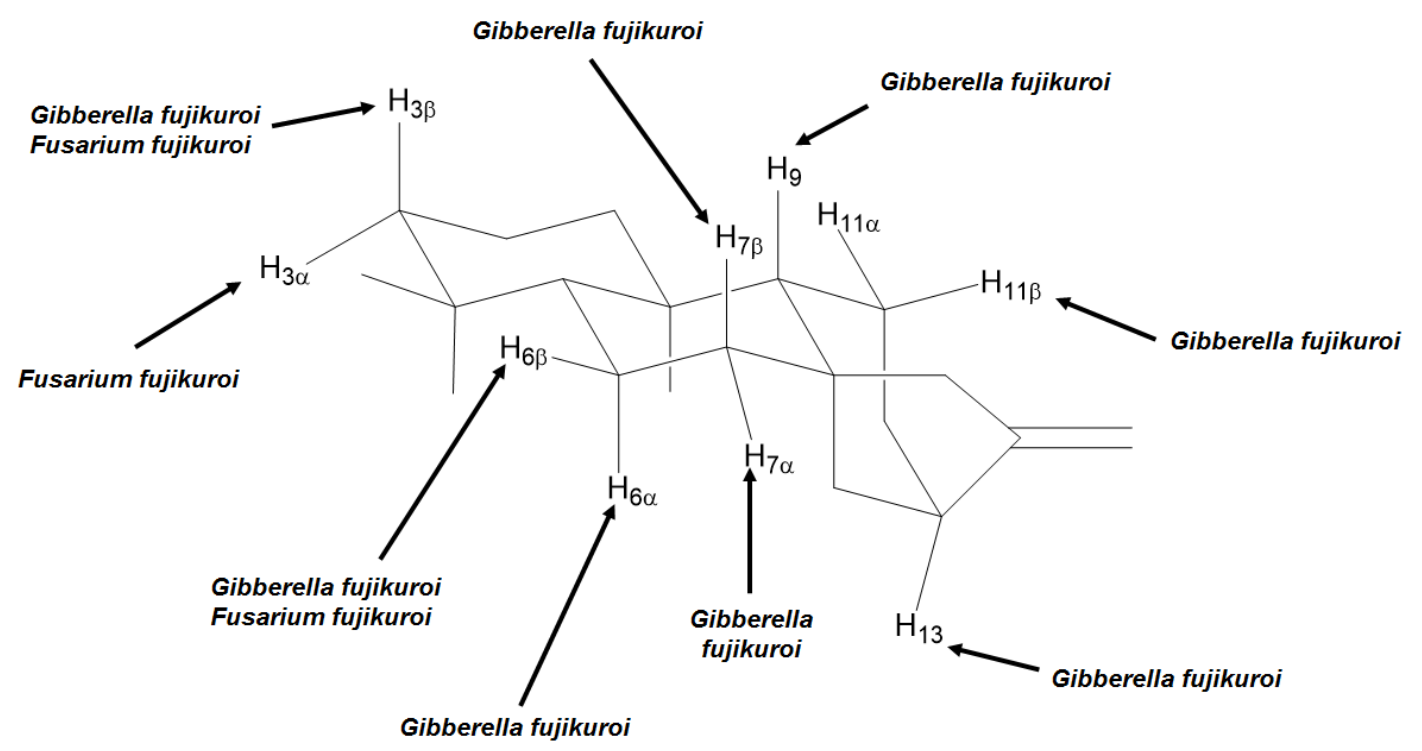

Since kaurane diterpenes are not xenobiotic to fungi from the G. fujikuroi complex, these biotransformations are classified as biosynthetically directed biotransformations. The use of inhibitors of the natural biosynthesis is very common in this kind of experiments to avoid production of natural metabolites that otherwise may be confused with biotransformation products. Concerning to diterpenes, biotransformations using G. fujikuroi is usually accompanied by the use of biosynthesis inhibitors like AMO 1618 (2-isopropyl-4-dimethylamino-5-methylphenyl-1-piperidinecarboxylate methyl chloride). This inhibitor acts inhibiting ent-kaurene biosynthesis however does not affect post-kaurene metabolism [58].

\section{Biotransformation Scope}

Biotransformation experiments are mostly performed in aqueous environments in order to achieve the optimum growth of the micro-organism. This feature makes biotransformations green processes, however it may also result in low yields for non-optimized processes. In typical fungal biotransformation experiments, the substrates are usually added to the culture medium as dilute solutions since high amounts of substrate can lead to growth inhibition or even death of the micro-organisms. Even when feeding dilute solutions to the fungi, high amounts of unaltered substrate is usually recovered. Products yields reported for initial non-optimized biotransformation experiments are typically below $10 \%$, although yields reaching $50 \%$ to $60 \%$ have also been related [59].

In most literature reports, it is not clear whether the product yield was calculated from the amount of substrate fed to the fungus or from the amount of substrate fed after adjusting for the amount of unconsumed starting material recovered from the experiments. It is a consensus that the aqueous environment and amount of substrate fed to the fermentation is an important issue in biotransformation yields [1]. Isolation of biotransformation products is sometimes only possible after derivatization, like esterification of carboxyl groups. 
Two stage cultures seems to have been more frequently used [31] than direct inoculation of biotransformation media. In two stage cultures, the fungus is firstly grown in the liquid medium (stage 1) and then transferred to a vessel containing fresh sterile liquid medium (stage 2), where the biotransformation will take place after further mycelia development, followed by substrate addition. In a number of successful biotransformation experiments, incubation times varied from 6 [60] to 14 days $[23,26,28]$, although smaller or longer incubation times can be found. Shorter incubation time seems to be very suitable for biosynthetically directed biotransformations $[53,61]$.

The literature shows that, in some cases, very short biotransformation periods can also result in products like the biotransformation of the diketone ent-18-acetoxykaur-15-ene-3,7-dione (7) by the fungus Curvularia lunata that furnished the product ent-17,18-dihydroxykaur-15-ene-3,7-dione (8) in very high yield (58\%) after only $30 \mathrm{~h}$ [62]. In the same way, biotransformation of ent-18-acetoxykaur15-en-7-one (9) with the fungus $C$. lunata furnished the product ent-17,18-dihydroxykaur-15-en-7-one (10) in $18 \%$ yield after only $48 \mathrm{~h}$ (Scheme 1) [62].

Scheme 1. Biotransformation of ent-18-acetoxykaur-15-ene-3,7-dione (7) and ent-18acetoxykaur-15-en-7-one (9) by the fungus $C$. lunata.

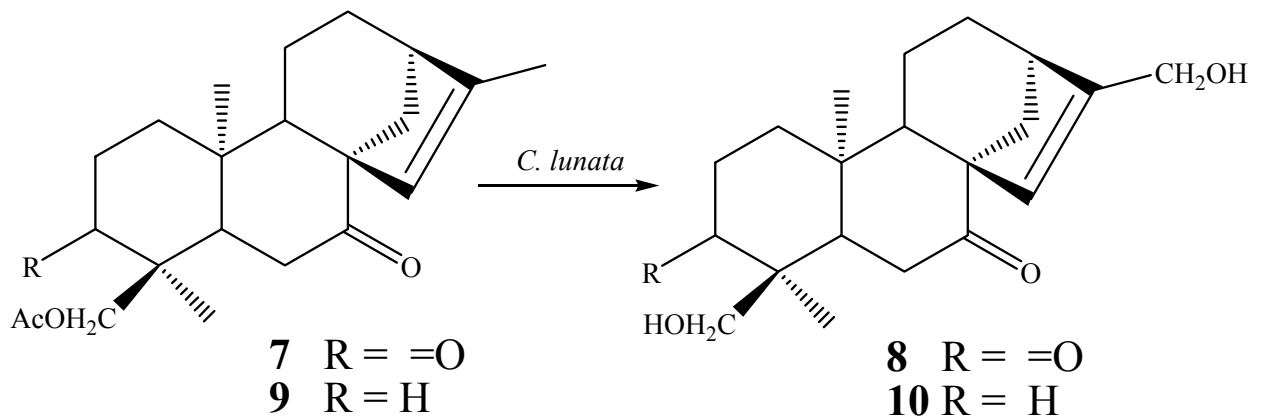

The nomenclature of kaurane diterpenes used in this review follows the guidelines described by Giles [63]. The prefix ent- before the name of the compounds means complete inversion at all configurations stated in relation to the fundamental parent structure.

\section{Most Common Methylene Hydroxylation in Kaurane Diterpenes}

The most common and also more attractive transformation carried out by fungi on kaurane structures is hydroxylation. This is of great value due to the possibility of achieving increased biological activities, as explained before. The following examples will illustrate that nearly all carbons of the kaurane skeleton have been activated with hydroxyl groups by the action of filamentous fungi.

Some of the most common hydroxylations of kaurane diterpenes seem to take place in carbons 7 and 11, either in the $\alpha$ - or $\beta$-positions. Carbons 3,6,9, and 13 have been hydroxylated less frequently by biotransformation, however these are also commonly described as hydroxylation sites. For instance, from a total of 65 randomly chosen papers about biotransformations of kaurane diterpenes (Table 2), 56 reported mostly hydroxylation of positions 7 (82.1\%), 11 (41.1\%), $6(23.2 \%), 13(14.3 \%), 3$ and 9 (12.5\% each), respectively. It is not uncommon to find examples in which both positions 7 and 11 were simultaneously hydroxylated. For instance, the biotransformation of ent-3 $\beta, 15 \alpha$-dihydroxy-kaur-

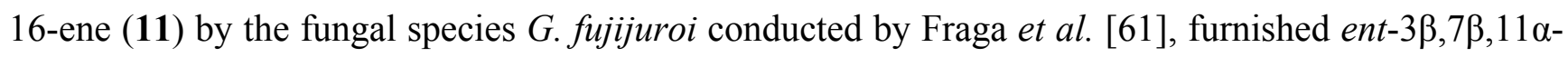


trihydroxy-15-oxo-kaur-16-ene (12) (Scheme 2). Regarding the $\alpha$ - or $\beta$-orientation of the hydroxyl groups introduced in the molecules via fungal biotransformations, the $\beta$-orientation seems to be prefered, especially with regard to carbons 7 and 11 . This may be result of the steric hindrance caused by carbons 18 and 20, both located on the $\alpha$-face of kaurane compounds.

Table 2. Examples of fungi able to hydroxylate kaurane diterpenes, positions and stereochemistry of hydroxylations.

\begin{tabular}{ccc}
\hline Fungal species & $\begin{array}{c}\text { Positions and } \\
\text { stereochemistry }\end{array}$ & References \\
\hline Absidia blakesleeana & $7 \beta, 11 \alpha, 13$ & {$[64]$} \\
Aspergillus niger & $3 \alpha, 7 \beta, 11 \alpha$ & {$[23,27,65]$} \\
Aspergillus ochraceus & $6 \beta, 7 \alpha, 13$ & {$[66,67]$} \\
Calonectria decora & $7 \alpha, 7 \beta$ & {$[66,67]$} \\
Cephalosporium aphidicola & $3 \alpha, 11 \beta$ & {$[68-70]$} \\
Cunninghamella bainieri & 9 & {$[43]$} \\
Cunninghamellha & $7 \beta$ & {$[71]$} \\
blakesleeana & $3 \alpha, 3 \beta, 6 \beta, 7 \beta$ & {$[60]$} \\
Fusarium fujikuroi & $11 \beta$ & {$[72]$} \\
Fusarium moniliforme & $13,6 \alpha, 7 \alpha, 7 \beta, 9,11 \beta$, & {$[10,53,55-58,61,73-100]$} \\
Gibberella fujikuroi & $6 \alpha, 7 \alpha, 9,11 \beta$ & {$[21,101,102]$} \\
Mucor plumbeus & $7 \beta, 11 \alpha, 11 \beta$ & {$[23]$} \\
Mucor recurvatus & $11 \beta$ & {$[103]$} \\
Psylocybe cubensis & $3 \alpha, 7 \alpha, 7 \beta, 13$ & {$[59,62,66,67]$} \\
Rhizopus nigricans & $7 \beta, 9$ & {$[64]$} \\
Rhizopus oligosporus & $7 \beta, 9,11 \beta$ & {$[31]$} \\
Rhizopus stolonifer & $7 \alpha, 7 \beta, 11 \beta$ & \\
Verticillium lecanii & &
\end{tabular}

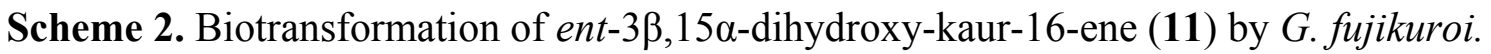

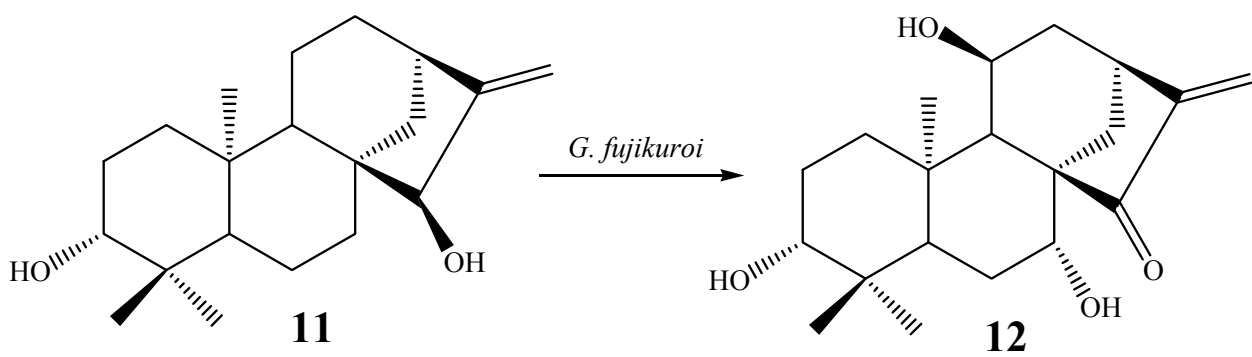

In addition to the positions and orientations mostly functionalized by biotransformation, another point of interest is the fungal species most employed in biotransformations of kaurane diterpenes. Table 2 presents the great variety of fungi employed in these reactions, the hydroxylation site and stereochemistry of the hydroxyl group introduced in the molecule. It can be noticed that some of these biotransformations consist of regioselective introduction of hydroxyl groups. Many other examples are available in the literature and some of them will also be discussed later in this review. 
It is interesting to observe that some species are able to hydroxylate the same carbon both on the $\alpha$ and $\beta$-faces, such as carbon 3 by F. fujikuroi, or carbon 6 by G. fujikuroi, among other (Figure 5).

Figure 5. Examples of fungi able to hydroxylate both $\alpha$ and $\beta$ positions of the same carbon of kaurane diterpenes.

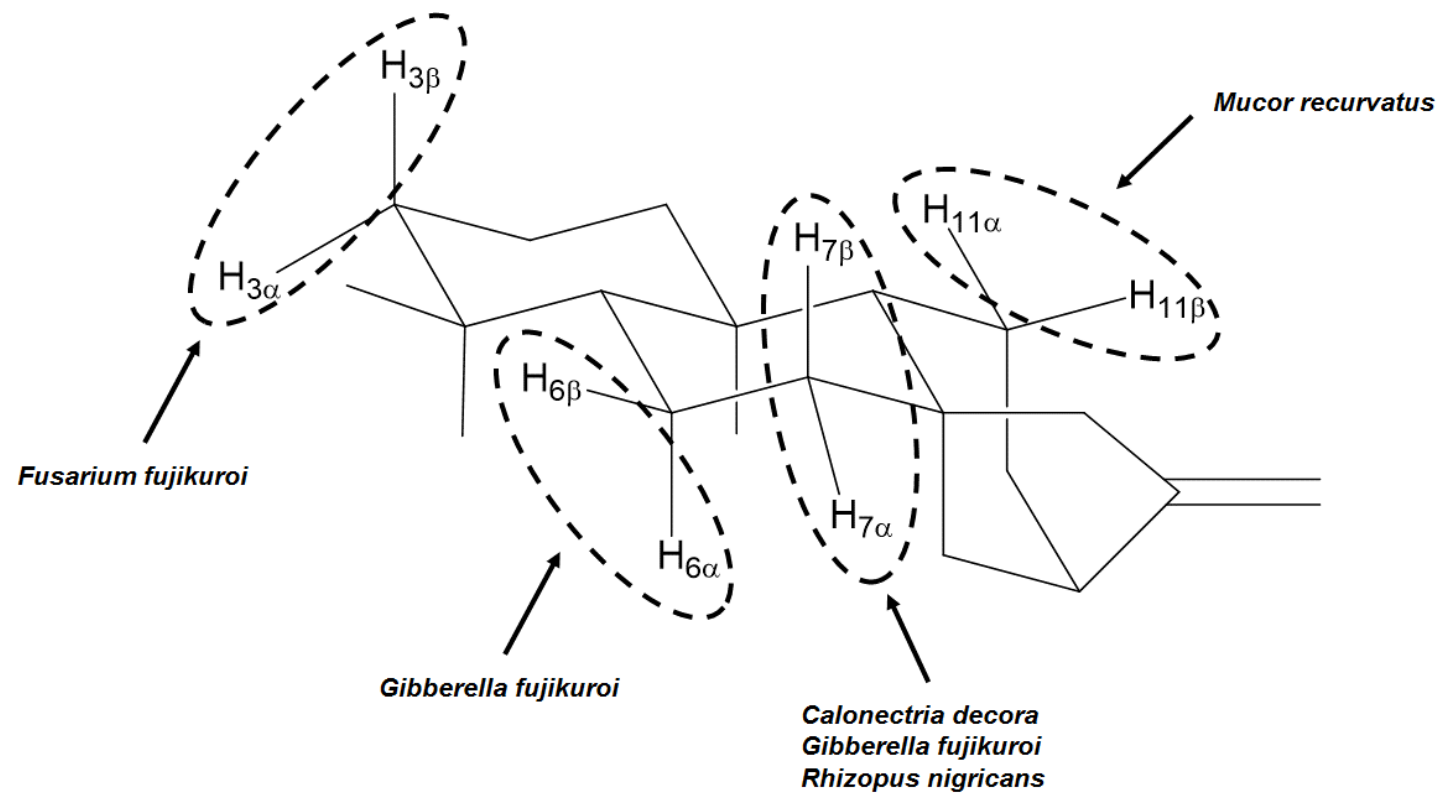

Some fungi have the ability of performing more than one hydroxylation in different positions of the starting materials. In this way, the biotransformation of ent-18-acetoxykaur-15-en-7-one by $R$. nigricans furnished the product 13 [59], while ent-15-oxo-kaur-16-ene led to the isolation of derivative 14 after biotransformation with G. fujikuroi [58]. In another example, ent-15ß-hydroxykaur-9 (11),16-dien-19-oic acid [60] was biotransformed in the derivative 15 by F. fujikuroi (Figure 6).

Figure 6. Chemical structures of hydroxylated derivatives 13-15, obtained by the action of R. nigricans and G. fujikuroi on kaurane skeleton.
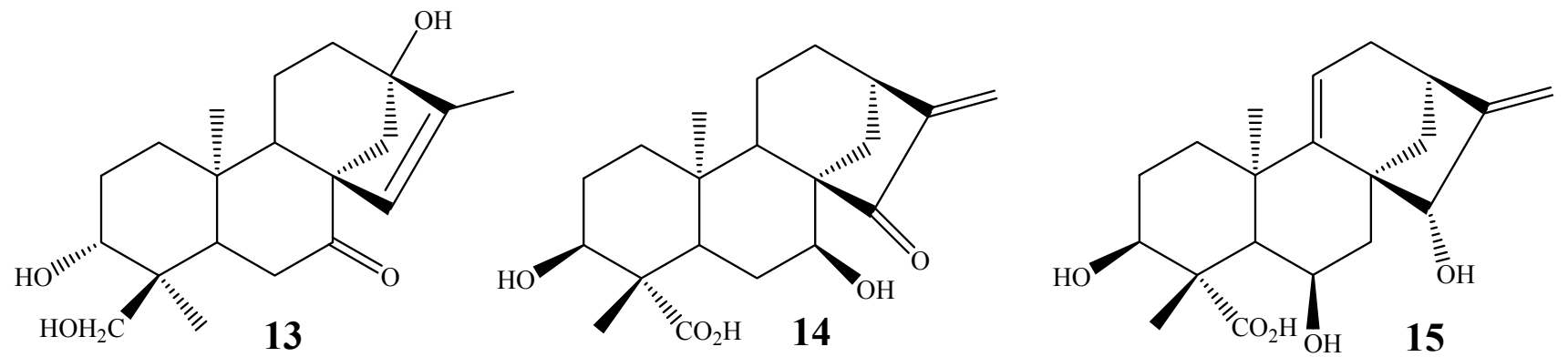

G. fujikuroi can produce two vicinal hydroxylations on ring B to furnish vic- $\beta, \beta-[75,86,95]$ and $\beta, \alpha$-diols (Figure 7) [61]. 
Figure 7. Chemical structures of the $\beta, \beta$ and $\beta, \alpha$ kaurane vic-diols 16-19.

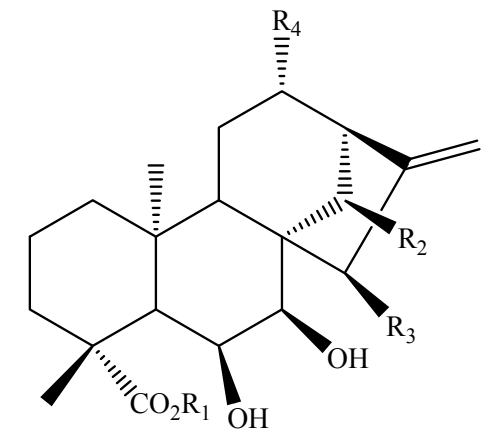

$16 \mathrm{R}_{1}=\mathrm{H} \quad \mathrm{R}_{2}=\mathrm{H} \quad \mathrm{R}_{3}=\mathrm{OH} \quad \mathrm{R}_{4}=\mathrm{H}$

$17 \mathrm{R}_{1}=\mathrm{H} \quad \mathrm{R}_{2}=\mathrm{H} \quad \mathrm{R}_{3}=\mathrm{H} \quad \mathrm{R}_{4}=\mathrm{OH}$

$18 \mathrm{R}_{1}=\mathrm{CH}_{3} \quad \mathrm{R}_{2}=\mathrm{OH} \quad \mathrm{R}_{3}=\mathrm{H} \quad \mathrm{R}_{4}=\mathrm{H}$

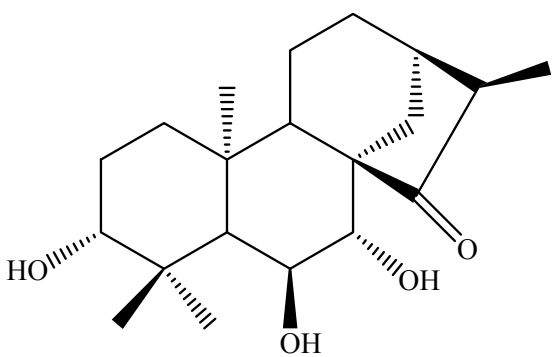

19

The position $\mathrm{C}-7$ is one of the most hydroxylated positions, regardless the fungal species used, as shown in Figure 8.

Figure 8. Examples of fungi able to carry on hydroxylation in carbon 7 of the kaurane skeleton.

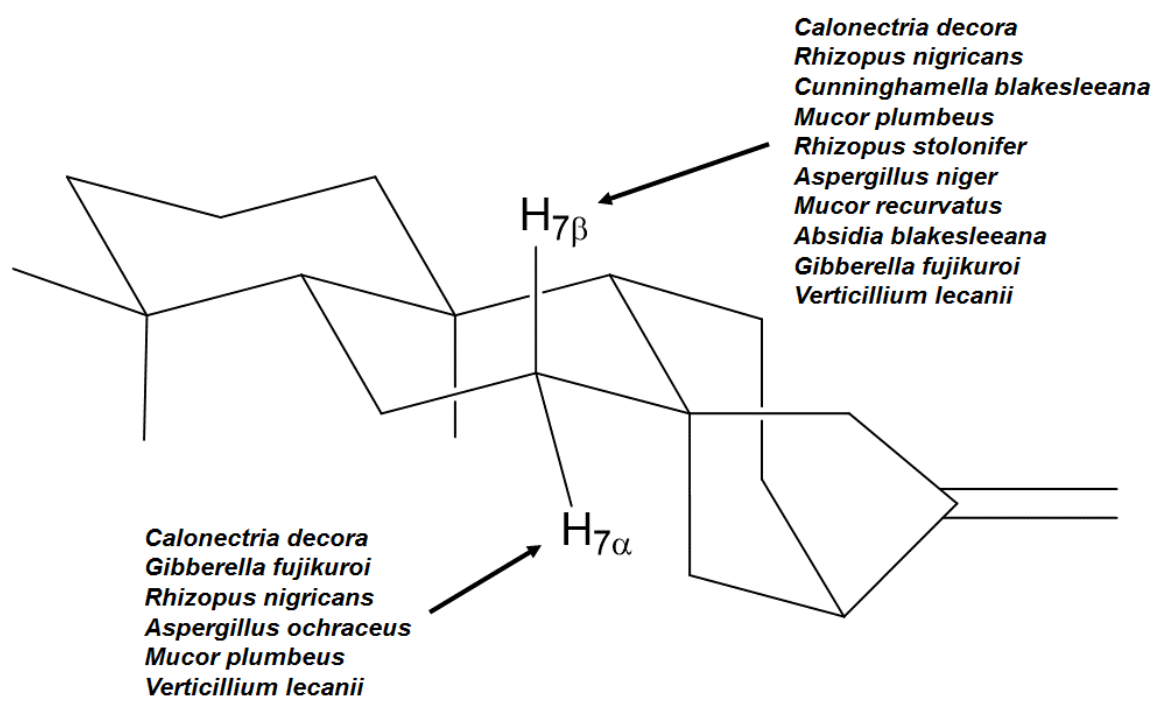

Shigematsu et al. [84], and Fraga et al. [92,96,100] using G. fujikuroi, isolated a great diversity of C-7 and C-11 dihydroxylated products 20-31 (Figure 9).

Boaventura et al. [101], working with M. plumbeus, Marquina et al. [27] using A. niger and Taveepanich et al. [64] using the fungus A. blakesleeana also isolated 7,11 dihydroxylated products 32, 33, showing that this behavior is not unique to G. fujikuroi (Figure 10).

Some variations are found, as, for example, Fraga et al. [61] reported the biotransformation of ent-3 $\beta$-hydroxy-15-oxo-(16S)-kaurane (34) with the isolation of products hydroxylated in positions 6 and 11 (35) by biotransformation using G. fujikuroi, while Taveepanich et al. [64] also isolated a dihydroxylated product at positions 7 and 13 (36) of kaurenoic acid with the fungus $A$. blakesleeana (Figure 11). 
Figure 9. Chemical structures of the great structural diversity of biotransformation products hydroxylated in carbons 7 and 11 by the fungus G. fujikuroi.
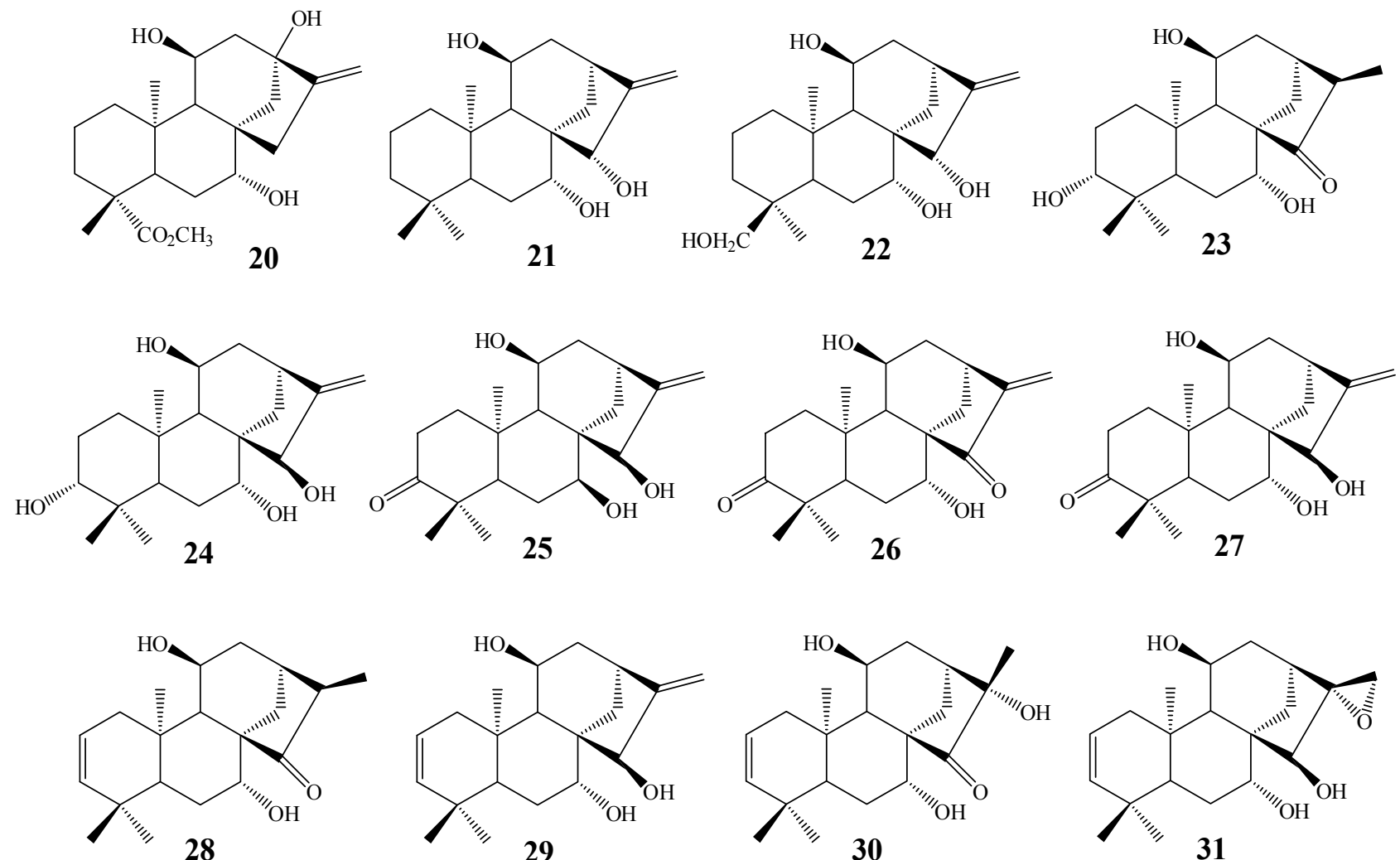

Figure 10. Chemical structures of products $\mathbf{3 2}$ and $\mathbf{3 3}$.
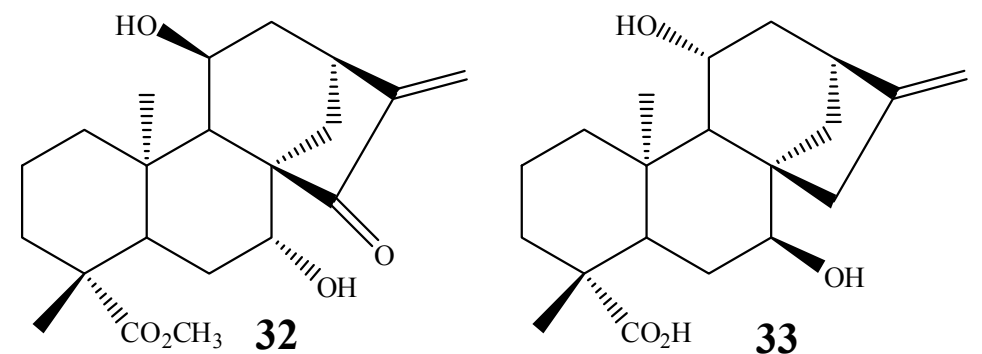

Figure 11. Chemical structures of starting material 34 and products hydroxylated in $\mathrm{C}-6 / \mathrm{C}-$ 11 and C-7/C-13 by the fungi G. fujikuroi and A. blakesleeana.
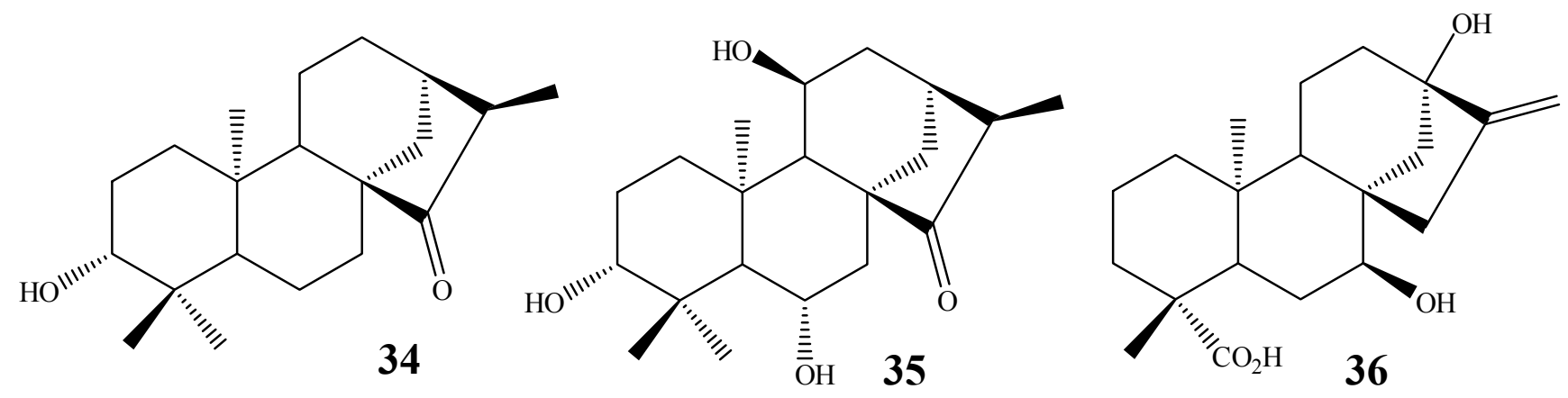
Table 3 gives an interesting comparison of the yields obtained by two groups, working with the same fungi and closely related starting materials $[66,67]$.

Table 3. Comparison on the yields obtained by Beilby et al. [66] and Ghisalberti et al. [67].

\begin{tabular}{|c|c|c|c|c|}
\hline Substrate & Fungus & Positions hydroxylated & Yield [66] (\%) & Yield [67] (\%) \\
\hline \multirow{6}{*}{$\begin{array}{c}\text { ent-19-hydroxy-16-oxo- } \\
\text { 17-nor-kaurane }\end{array}$} & A. ochraceus & $7 \alpha$ & n.i. & 20 \\
\hline & \multirow{2}{*}{ C.decora } & $1 \alpha$ & 10 & 10 \\
\hline & & $7 \alpha$ & 10 & 10 \\
\hline & \multirow{3}{*}{ R. nigricans } & $1 \alpha$ & 20 & 20 \\
\hline & & $7 \alpha$ & 20 & 20 \\
\hline & & & & \\
\hline \multirow{5}{*}{$"=\mathrm{CO}_{2} \mathrm{H} \quad 38$} & A. ochraceus & 13 & 5 & 5 \\
\hline & \multirow{3}{*}{ C. decora } & $1 \alpha$ & 10 & 5 \\
\hline & & $7 \alpha$ & 15 & 15 \\
\hline & & $7 \beta$ & 40 & 40 \\
\hline & \multirow{3}{*}{ R. nigricans } & $1 \alpha$ & 30 & 30 \\
\hline \multirow{2}{*}{$\begin{array}{l}\text { ent-16-oxo-17-nor- } \\
\text { kauran-19-oic acid }\end{array}$} & & $7 \alpha$ & 30 & 30 \\
\hline & & $7 \beta$ & 5 & 5 \\
\hline \multirow{6}{*}{$\begin{array}{l}\text { " } " \mathrm{CO}_{2} \mathrm{H} \quad 1 \\
\text { ent-kaur-16-en-19-oic } \\
\text { acid }\end{array}$} & A. ochraceus & $16 \alpha, 17$ & 20 & 10 \\
\hline & \multirow{3}{*}{ C. decora } & $7 \alpha, 15 \alpha$ & 30 & 30 \\
\hline & & $15 \alpha$ & 5 & 5 \\
\hline & & $7 \alpha$ & 5 & 5 \\
\hline & \multirow{2}{*}{ R. nigricans } & $16 \alpha, 17$ & n.i. & 10 \\
\hline & & $7 \beta$ & 25 & 25 \\
\hline \multirow{6}{*}{$\begin{array}{l}\text { ent-3 } \alpha \text {-hydroxy-16-oxo- } \\
\text { acid }\end{array}$} & \multirow{2}{*}{ A. ochraceus } & $6 \beta$ & 30 & n.i. \\
\hline & & $7 \alpha$ & 25 & n.i. \\
\hline & C. decora & $7 \alpha$ & 40 & n.i. \\
\hline & \multirow{3}{*}{ R. nigricans } & $1 \alpha$ & 25 & n.i. \\
\hline & & $7 \alpha$ & 35 & n.i. \\
\hline & & & & \\
\hline
\end{tabular}

\section{Hydroxylations on Ring D}

One of the most often reported structural changes accomplished by filamentous fungus on the ring $\mathrm{D}$ of kaurane diterpenes is the modification of the double bond between carbons 16 and 17, present in most of the compounds subjected to biotransformations. Incubation of epi-candol A (ent-7 $\beta$-hydroxykaur-16-ene, 40), with the fungus $G$. fujikuroi led to the formation of the diol ent-7 $\beta, 16 \beta, 17$ trihydroxy-kaur-16-ene (41) in $0.45 \%$ yield (Scheme 3 ) [53].

Other microorganisms also perform the oxidation of the exocyclic double bond. R. stolonifer was able to transform ent-kaurenoic acid (1) in the compound ent-16ß,17-dihydroxy-kauran-19-oic acid (42) in a yield of $4 \%$. The C-16 hydroxylation by the fungus $R$. stolonifer, as well as that performed by G. fujikuroi, occurred both in $\alpha$-position (Scheme 3) [104]. 
Scheme 3. Hydroxylation of carbons 16 and 17 by fungi G. fujikuroi and R. stolonifer.

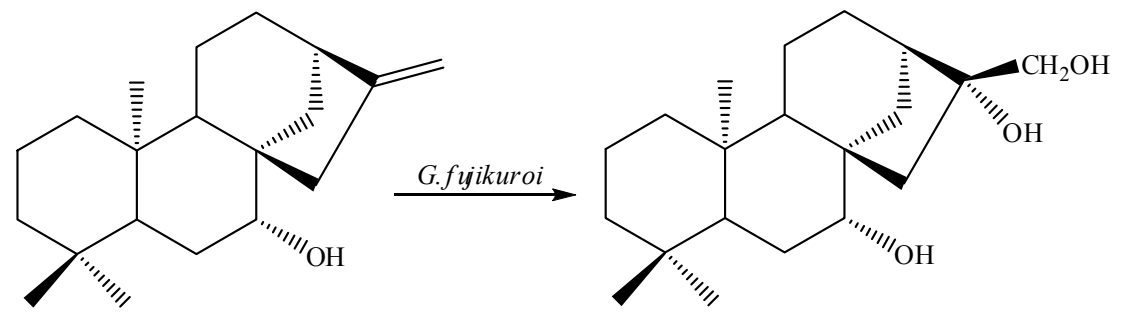

40

41

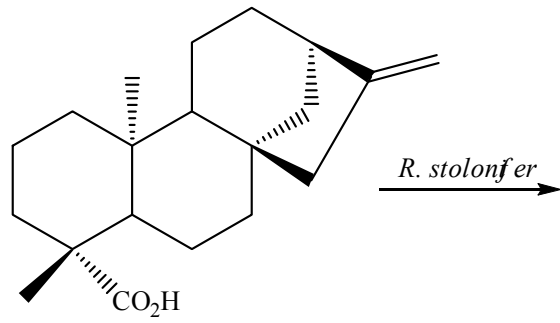

1

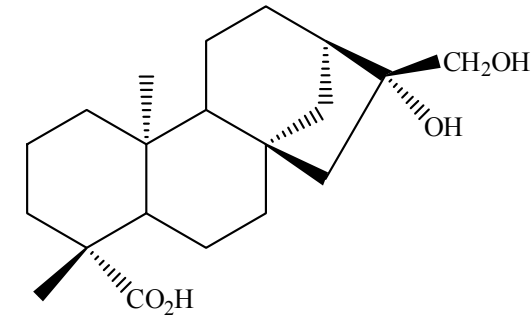

42

Fraga et al. [60] showed a route involving compounds 43-46 in the biotransformation of kaurane derivatives with the fungus $F$. fujikuroi. The dihydroxylation of the positions 16 and 17 in

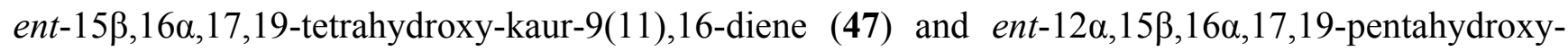
kaur-9(11),16-diene (48) is derived from the formation of an epoxide with subsequent hydration of this group (Scheme 4). Fraga et al. proved that this last reaction is not performed by the fungus F. fujikuroi, but rather occurs naturally in the culture medium or during the purification procedure [60].

Scheme 4. Route proposed by Fraga et al. [60] for obtaining compounds 47 and 48.

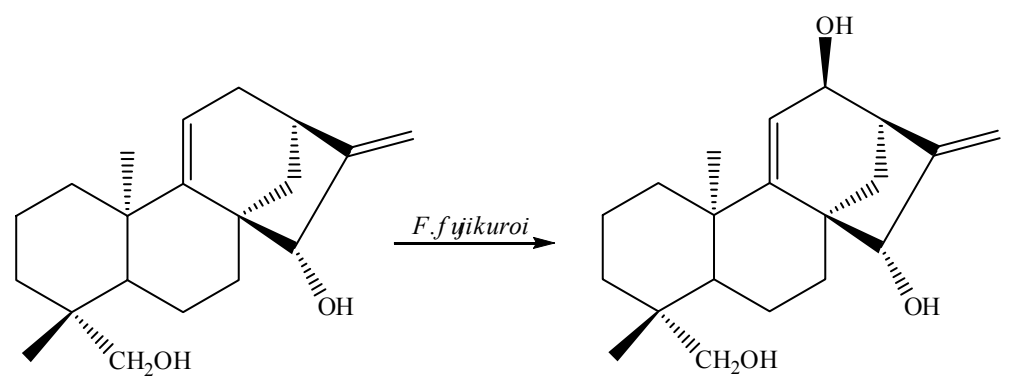

43

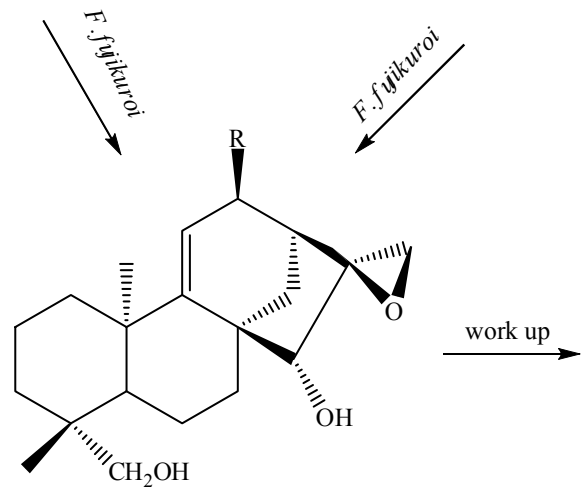

44

$45 \mathrm{R}=\mathrm{H}$

$46 \mathrm{R}=\mathrm{OH}$

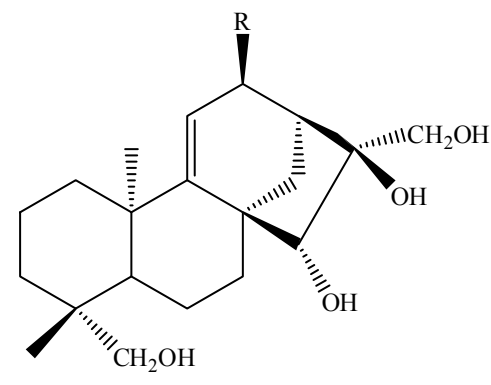

$47 \mathrm{R}=\mathrm{H}$

$48 \mathrm{R}=\mathrm{OH}$ 
The proposal of a mechanism involving an epoxide at $\mathrm{C}-16 / \mathrm{C}-17$ as intermediary in the biotransformation is corroborated by several other studies reporting epoxidation by fungi [62,89]. For instance, Fraga et al. [58] reported the action on G. fujikuroi on the substrate ent-7-oxo-18-hydroxykaur-16-ene (49) recovering an epoxide, ent-18-hydroxy-16 $\beta, 17$-epoxy-7-oxo-kaurane (50) (0.36\% yield), in a stereoselective biotransformation (Scheme 5).

Scheme 5. Epoxide formation by G. fujikuroi.

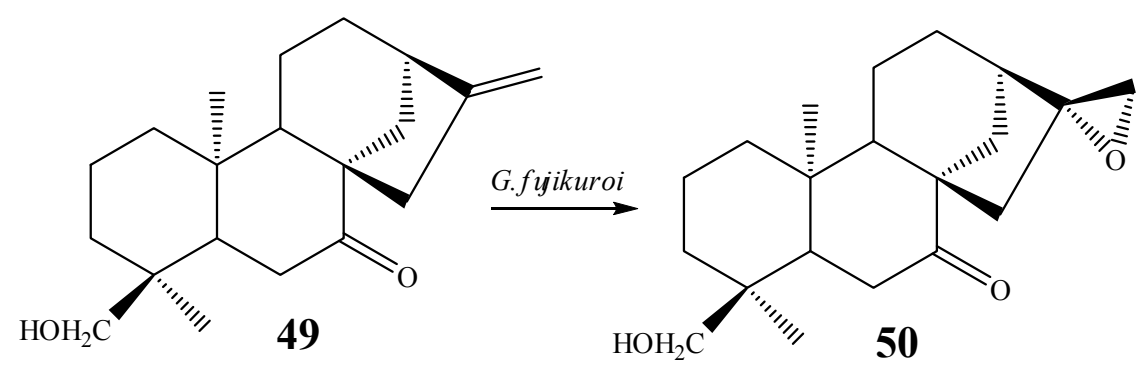

The fungal action in the exocyclic double bond of kaurane diterpenes does not necessarily lead to diols. Rocha et al. [28] reported a good example of mono- and dihydroxylation of the 16-17 double bond by the same fungal species. The biotransformation of the compound ent-kaur-16-en-19-ol with C. aphidicola was able to generate two products: ent-kauran-16 $\beta, 19$-diol (51, 0.54\%) and ent-kauran$16 \beta, 17,19$-triol $(52,1.86 \%)$. In both compounds, the hydroxyl group inserted in position 16 has the same $\alpha$-stereochemistry. Based on these results, it is possible that hydroxylation of C-17 ocurrs via the sequential hydroxylation of diol 51 [28]. Another C16-C17 diol bearing a 16a-hydroxyl group, ent-7 $\alpha, 16 \beta, 17,18$-tetrahydroxy-kaurane (53), has been isolated from the biotransformation of epicandicandiol by M. plumbeus (Figure 12) [102].

Figure 12. Chemical structures of compounds 51-53.
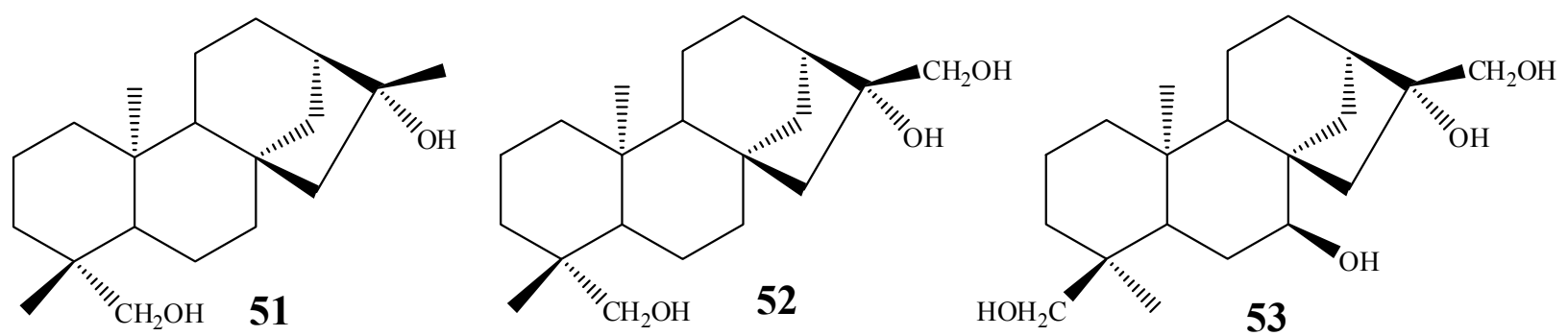

Allylic hydroxylation at C-15 of kaurane diterpenes is another structural modification that has also been achieved by biotransformation. Introduction of a hydroxyl group at C-15 is one of the few reactions that also can be accomplished using chemical methods [106]. As an example, candicandiol (ent-7 $\beta, 18$-dihydroxy-kaur-16-ene, 54) was incubated with the fungus $M$. plumbeus, being hydroxylated at $\mathrm{C}-15 \alpha$ to furnish canditriol (55) as a minor metabolite (1.47\% yield) since a dihydroxylation product of (ent-7 $\beta, 16 \beta, 17,18$-tetrahydroxy-kaurane, 56) was isolated in higher yield (15.08\% yield) from the same experiment (Scheme 6) [102]. 
Scheme 6. Biotransformation of candicandiol (54) into 55 and 56 by M. plumbeus.

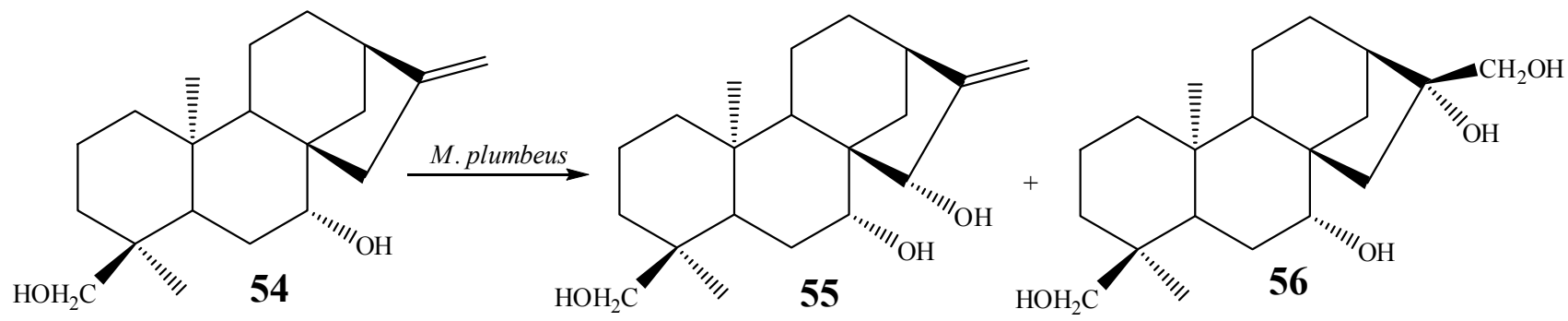

When comparing the yields of biotransformations performed with fungi of the genus Mucor [102] with other biotransformation experiments of kaurane diterpenes, it may be noticed that this species usually presents the best biotransformation yields. This genus is also reported to be efficient for the biotransformation of diterpenes with other skeletons [107,108].

\section{Some Unconventional Biotransformations}

The fungi have their own role in the hydroxylation of methylene groups. Comparing the literature available for fungal hydroxylation of, for instance, carbons 1, 11 and 12, all of them being methylene groups, $\mathrm{C}-1$ and $\mathrm{C}-11$ are spatially close to each other and subject to nearly the same spatial interactions, however, carbon 11 is much more frequently hydroxylated than carbon 1 . One of the factors that may explain the preferential hydroxylation of carbon 11 may be related to the fact that this hydroxylation is a step of the biosynthesis of some triterpenes and steroids, such as fusidic acid (57) (Figure 13), commonly produced by fungus. These compounds have some structural similarities with the skeletons of diterpenes [109].

Figure 13. Chemical structure of fusidic acid (57).

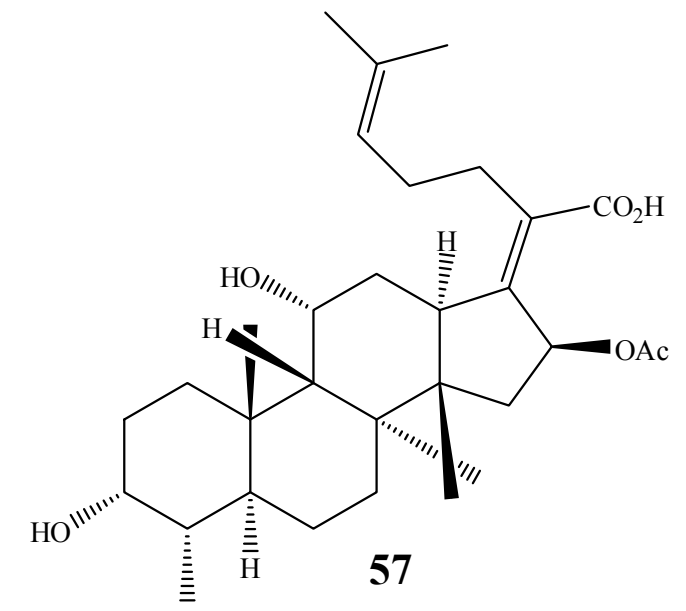

It has been reported that in the biotransformation of ent-kaur-16-ene derivatives by G. fujikuroi, a $15 \alpha$ - or $16 \alpha$-hydroxyl group, or a $15 \alpha, 16 \alpha$-epoxide group inhibits oxidation at C-19 and directs hydroxylation to $\mathrm{C}-11 \beta$ and $\mathrm{C}-7 \alpha[53,100]$.

A. niger is a fungal species known to introduce hydroxyl groups in kaurane diterpenes in positions C-7, C-11 [23,27], C-3 [65] and also at C-1, a non-conventional hydroxylation site. Kaurenoic acid, for instance, was incubated with $A$. niger to furnish the compound ent-1 $\beta, 7 \alpha$-dihydroxy-kaur-16-en-19-oic 
acid (58), bearing a hydroxyl group at C1 (5.8\% yield) (Figure 14) [27]. Other works also report biotransformations of kaurane diterpenes resulting in products with $\mathrm{C}-1$ hydroxylation by action of $A$. blakesleeana [64] and G. fujikuroi (compound 59) [100].

Figure 14. Chemical structures of kaurane diterpenes 58 and 59 bearing a C-1 hydroxyl group as a result of biotransformations.
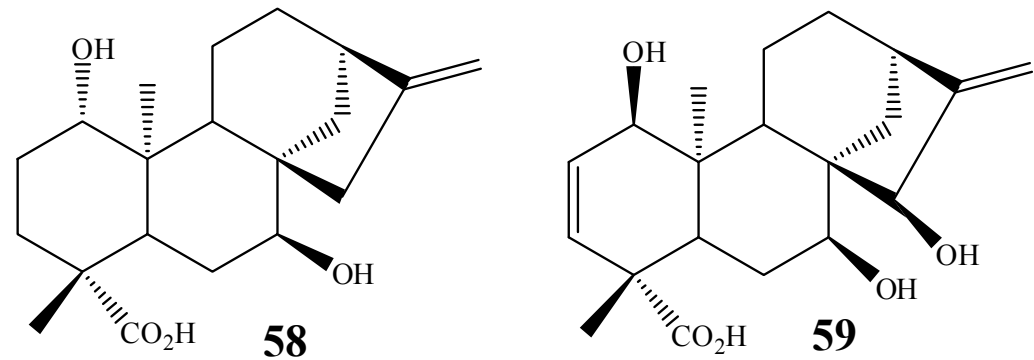

An interesting comparison concerns the $\mathrm{C}-11$ and $\mathrm{C}-12$ positions. Observing a tridimensional model of the kaurane molecule at an appropriate angle, the latter seems to be much more available for an enzymatic hydroxylation. However, C-12 has not been reported to be a common site for biotransformation.

Pechwang et al. [103] reported the biotransformation of kaurenoic acid by $P$. cubensis (magic mushroom). Mushrooms have not been frequently used as biocatalysts in biotransformations. $P$. cubensis, used in this experiment, is reported to possess hallucinogenic effects and its metabolic profile comprises the psychotropic substances psilocybin and psilocin [110-112]. P. cubensis was able to hydroxylate kaurenoic acid at C-12 producing 60 (8.3\% yield) (Figure 15) [103].

Hydroxylation of kaurane diterpenes at carbon 2 by biotransformation seems to have been first accomplished only in 2010 in the work of Rocha et al. [26]. In their work, ent-16-oxo-17-norkauran19-oic acid, obtained from kaurenoic acid, was biotransformed by $F$. proliferatum producing the C-2 hydroxylated derivative ent-2 $\alpha$-hydroxy-16-oxo-17-norkauran-19-oic acid (61), with a yield of 17.6\% (Figure 15).

Figure 15. Chemical structures of kaurane diterpenes bearing C-12 and C-2 hydroxylations.
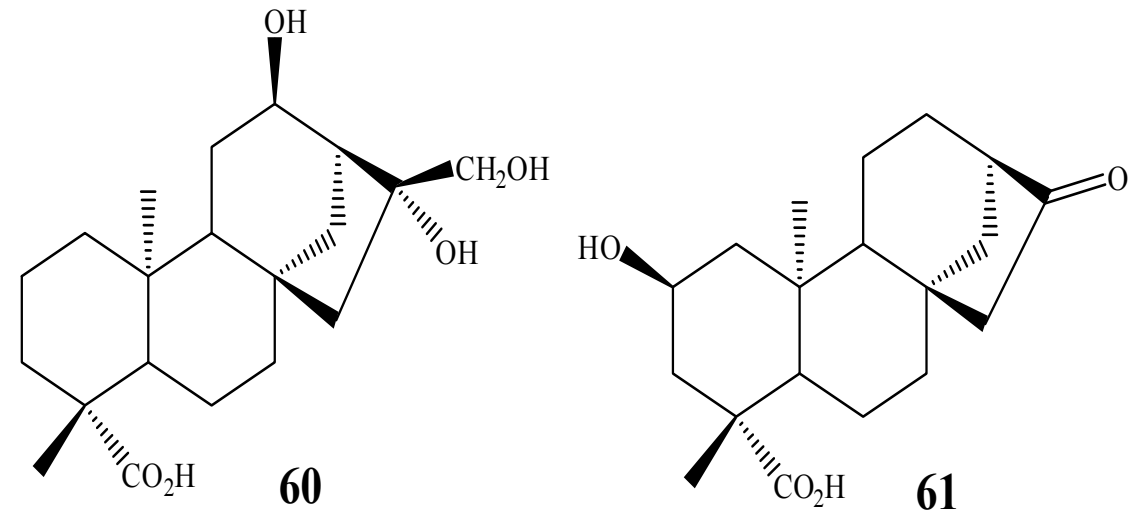

Later, Fraga et al. [10] reported that position C-2 could also be activated by the biotransformation of ent-7 $\alpha$-acetoxy-kaur-16-ene by G. fujikuroi. Fraga et al. proposed a biotransformation pathway from 
a sequence of oxidations that resulted in the formation of the product hydroxylated in C-2. The proposal is based on the products 62-67 obtained from their experiment and is shown in Scheme 7 [10].

Scheme 7. Route proposed by Fraga et al. for obtaining the compound ent-7 $\alpha$-acetoxy$2 \alpha, 3 \alpha$-dihydroxy-kaur-16-en-19-oic acid (67) by biotransformation using G. fujikuroi.

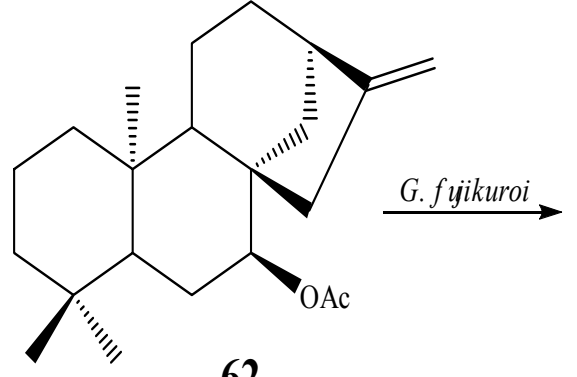

62

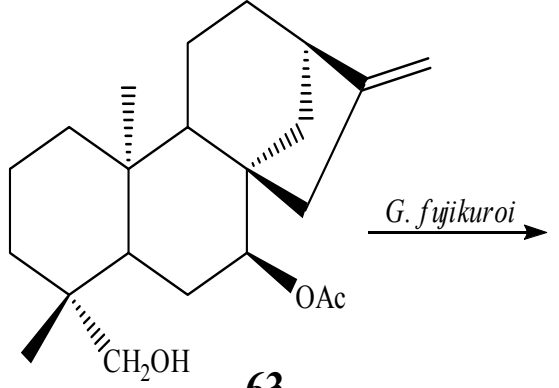

63

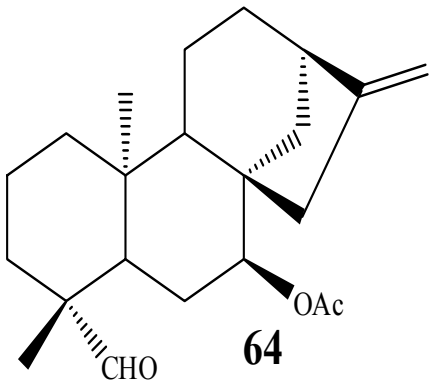

G. fyikuroi

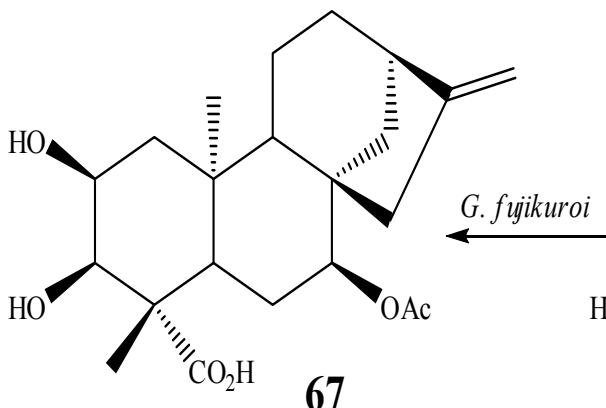

67
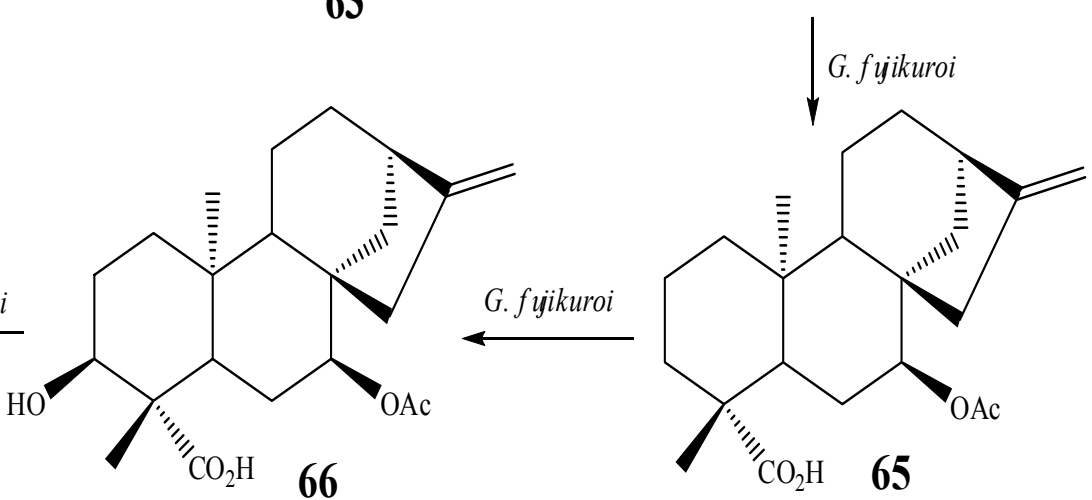

Biotransformation resulting in $\mathrm{C}-15$ allylic hydroxylation has been reported. The diterpene candicandiol, incubated with the fungus $M$. plumbeus produced the ent-7 $\beta, 15 \beta, 18$-trihydroxy-kaur-16ene (55) [102].

\section{Hydroxylations of Carbons 9 and 13}

Although most common fungal hydroxylations in kaurane diterpenes occur at methylene carbons, the methine carbons $\mathrm{C}-9$ and $\mathrm{C}-13$ have also been activated by biotransformations. On the other hand, C-5 still seems not to have been the target of microbial hydroxylation so far. In relation to the methine carbon C-9, Vieira et al. [105] reported the hydroxylation of methyl ent-17-hydroxy-16ßH-kauran-19oate by $R$. stolonifer. The product ent-9 $\alpha, 17$-dihydroxy-16S-kauran-19-oate (68) was isolated along with ent-7 $\alpha, 17$-dihydroxy-16 $\beta$-kauran-19-oate (12\% conversion each) (Figure 16). In the biotransformation of kaurenoic acid by $A$. blakesleeana and by $R$. oligosporus the same C-9 hydroxylated product, ent-7 $\alpha, 9 \alpha$-dihydroxy-kaur-16-en-19-oic acid (69), was obtained [64]. Hydroxylation at C-9 by biotransformation of candicandiol by $M$. plumbeus producing the C-9 hydroxylated compound ent-7 $\beta, 9 \alpha, 18$-trihydroxy-kaur-16-ene (70) was also reported [102]. R. stolonifer is known to produce hydroxylation at position C-9 in steviol methyl ester to afford methyl ent-9 $\alpha, 13$-dihydroxykaur-16-en19-oate (71) [90]. 
Figure 16. Chemical structures of some biotransformation products hydroxylated at C-9.

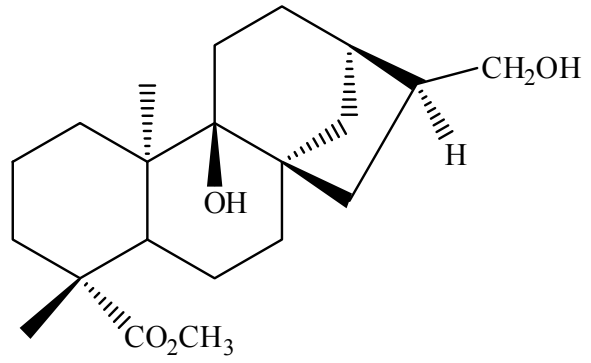

68

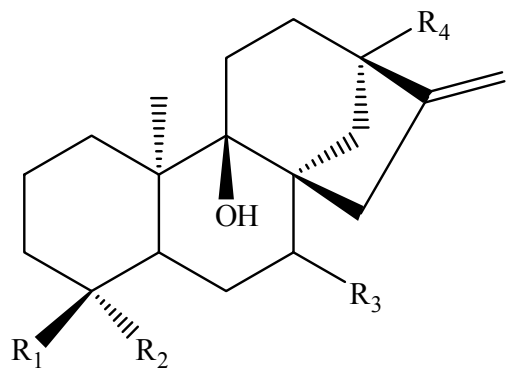

$69 \mathrm{R}_{1}=\mathrm{CH}_{3} \quad \mathrm{R}_{2}=\mathrm{CO}_{2} \mathrm{H} \quad \mathrm{R}_{3}=\beta-\mathrm{OH} \quad \mathrm{R}_{4}=\mathrm{H}$

$\begin{array}{llll}70 & \mathrm{R}_{1}=\mathrm{CH}_{2} \mathrm{OH} & \mathrm{R}_{2}=\mathrm{CH}_{3} & \mathrm{R}_{3}=\alpha-\mathrm{OH} \quad \mathrm{R}_{4}=\mathrm{H}\end{array}$

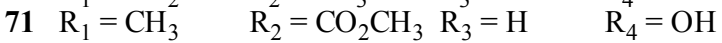

The fungus $R$. nigricans was able to insert a C-13 hydroxyl group in the compound ent-18acetoxykaur-15-en-7-one in a regioselective biotransformation to furnish the compound ent-18acetoxy-13-hydroxykaur-15-en-7-one (72), in 27.83\% yield [62]. However, it seems to be more common to observe hydroxylation of $\mathrm{C}-13$ accompanied by hydroxylation of other carbons. For instance, the compound ent-7-oxo-kaur-16-ene, when incubated with G. fujikuroi, furnished the diol

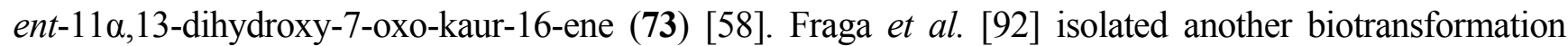
product 74 with C-11 and C-13 hydroxylation produced by the fungus G. fujikuroi. In biotransformation of kaurenoic acid by $A$. blakesleeana hydroxylation of $\mathrm{C}-13$ was accompanied by introduction of a $7 \alpha-\mathrm{OH}$ into the product, to give ent-7 $\beta, 13$-dihydroxy-kaur-16-en-19-oic acid (75) (Figure 17) [64].

Figure 17. Products hydroxylated at positions C-11 and C-13.
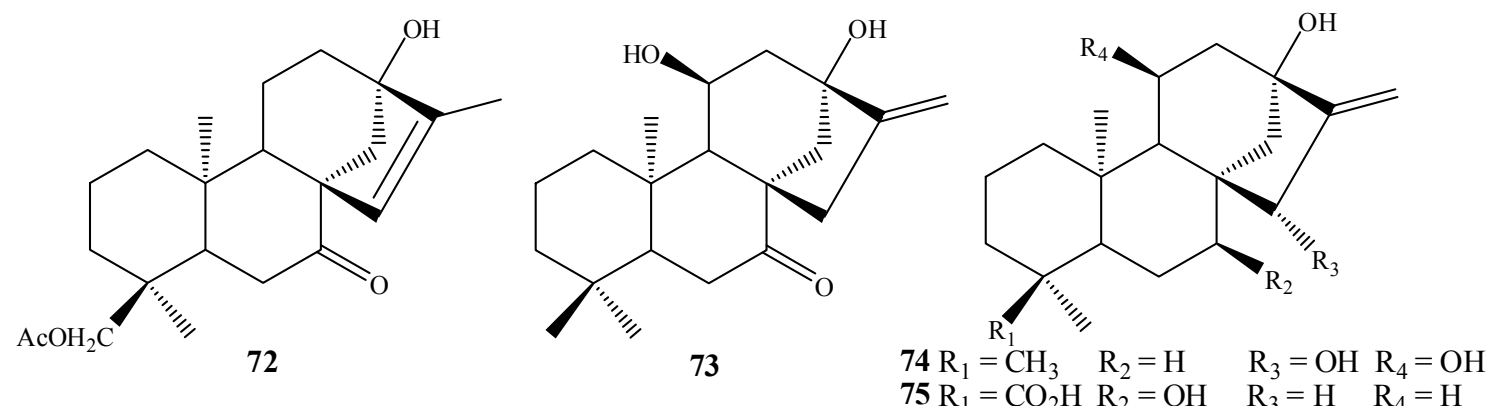

\section{Hydroxylation of Kaurane Diterpene Methyl Groups}

Kaurane diterpenes often possesses C-18 and C-20 and eventually C-17 as non-functionalized methyl groups [113,114] although is more common to find a double bond between C16 and C-17. Position 19 may be oxidized as a carboxyl group or a primary alcohol. Considering the four possible methyl groups on the carbon skeleton, the methyl groups C-19 and C-20 are the more sterically hindered because they experience 1,3-diaxial interactions between each other. The C-18 methyl group is in an equatorial position, and therefore, in theory, it should be more available to enzymatic attack.

In relation to the presence of a C-17 methyl group attached to $\mathrm{C}-16$, in theory, a $16 \alpha-\mathrm{CH}_{3}$ should be more exposed. In the $\alpha$-position, this methyl group suffers less hindrance than a $16 \beta-\mathrm{CH}_{3}$ since the latter is close to the $\mathrm{C}-11$ methylene group, considering ring $\mathrm{C}$ in the preferential chair conformation. 
However, microbial hydroxylation relies more on the microorganism's features and most of them can overcome steric hindrance. For instance, hydroxylation at C-19 was achieved by incubating ent-7 $\alpha$-acetoxy-kaur-16-ene (62) with $G$. fujikuroi to furnish ent-7 $\alpha$-acetoxy-19-hydroxy-kaur-16-ene (63) $(1.26 \%$ yield) [10]. When a kaurane derivative hydroxylated at the C-18 methyl group (ent-7 $\alpha$ acetoxy-18-hydroxy-kaur-16-ene, 76) was used as substrate in a biotransformations experiment using the same fungus, hydroxylation at C-19 was also achieved, leading to the formation of ent-7 $\alpha$-acetoxy18,19-dihydroxy-kaur-16-ene (77), in 2.14\% yield (Scheme 8) [10].

Scheme 8. Hydroxylation of methyl C-19 by G. fujikuroi.
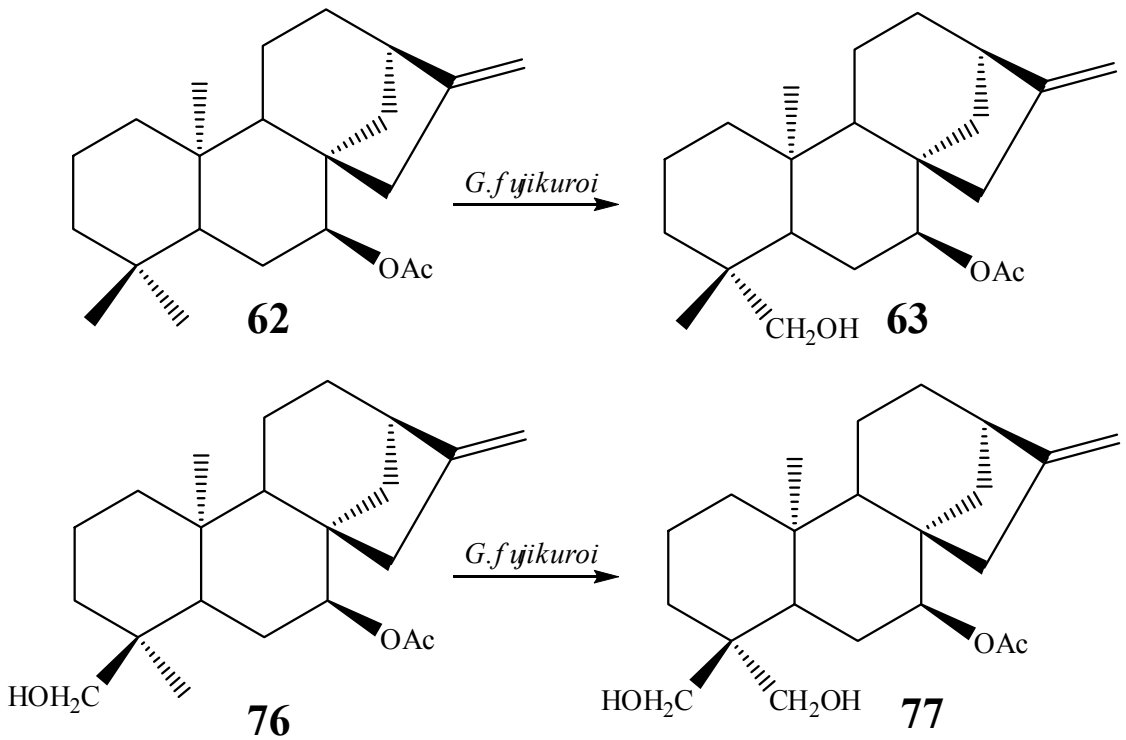

In the studies with G. fujikuroi, the derivative containing a hydroxyl group at position 19 does not suffer a subsequent oxidation on carbon 18. Following the possible biosynthetic routes used by this species, it was noticed that after the hydroxylation of C-19, several oxidations occur, including hydroxylation at C-3 prior to hydroxylation at position 18 [10].

The incubation of candicandiol (54) with the fungus G. fujikuroi led to the isolation of a derivative hydroxyated at C-19 (ent-7ß,18,19-trihydroxy-kaur-16-ene, 78), in 4.5\% yield (Scheme 9) [53]. When the substrate used in this biotransformation contained an acetyl group at C-7, the yield of the C-19 hydroxylation was lower [10].

Scheme 9. Biotransformation of candicandiol by G. fujikuroi.

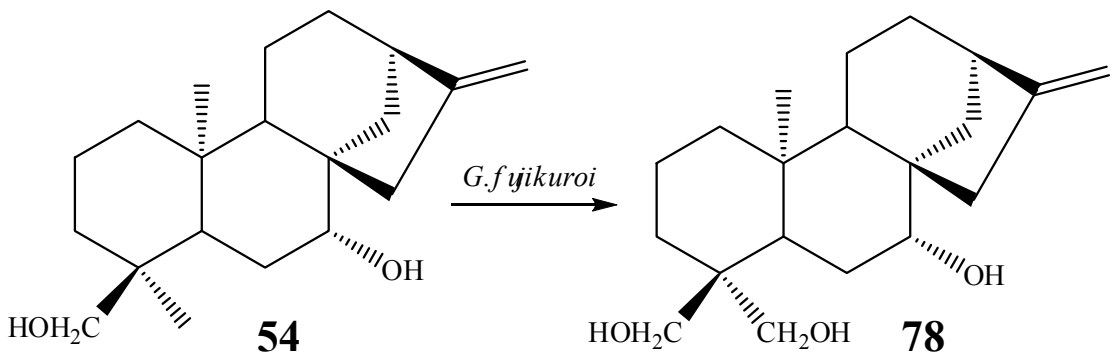


In another work, a substrate not functionalized at C-7 was used, and in this case it was possible to obtain the hydroxylation of methyl 18 when carbon 19 was hydroxylated. The authors proposed that the biotransformation of $\mathbf{7 9}$ by F. fujikuroi started with the hydroxylation at position C-6 $\beta$, based on the isolation of compound ent-6$\alpha, 15 \beta, 19$-trihydroxy-kaur-9(11),16-diene (80) from this experiment, followed by hydroxylation at $\mathrm{C}-18$ to furnish the product ent-6$\alpha, 15 \beta, 18,19$-tetrahydroxy-kaur9(11),16-diene (81, Scheme 10). Among the derivatives isolated from this biotransformation, the compound hydroxylated at C-19 was produced in lower yield and presented lower stability [60].

Scheme 10. Route proposed by Fraga et al. for the hydroxylation of carbon 18 .

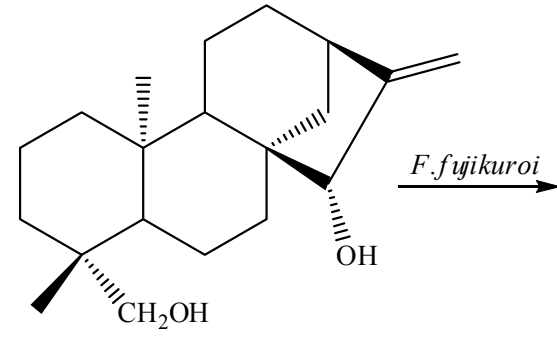

79

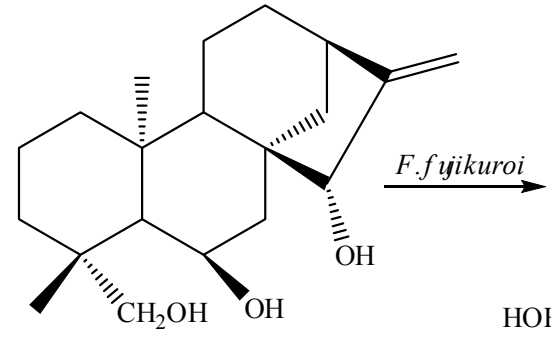

80

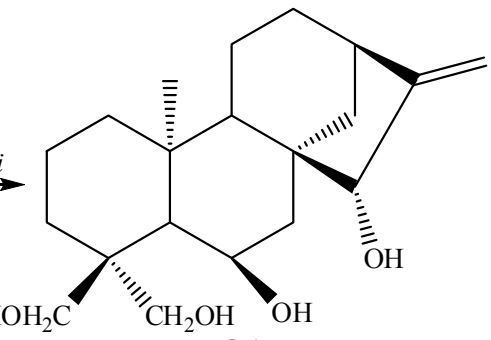

81

In an attempt to study the correlation between hydroxylation at C-18 and C-19 positions, Fraga et al . carried out a study using the compound ent-7-oxo-kaur-16-ene and derivatives containing different substitution patterns at C-3 and C-18 (compounds 82, 49, 85, Scheme 11). The compounds were incubated with the fungus G. fujikuroi [58].

Scheme 11. C-19 biotransformation using different products by G. fujikuroi.
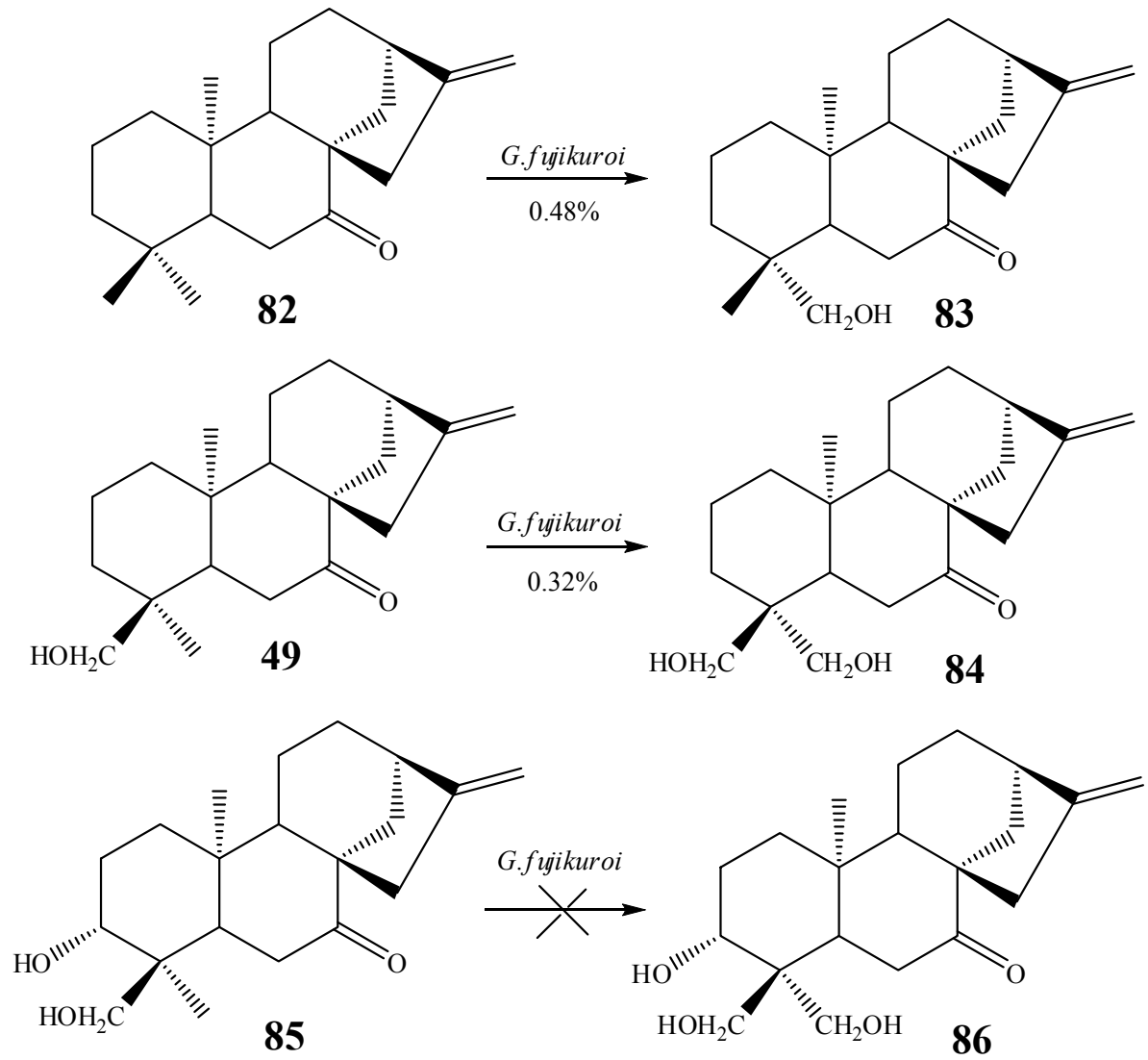
Compounds 83 and 84 were hydroxylated at C-19 while compound 86, a derivative hydroxylated at C-3, was not hydroxylated at C-19 by G. fujikuroi [58].

\section{Structural Alterations Other than Hydroxylation}

Hydroxylations are the structural modifications most often reported as a result of biotransformations of kaurane diterpenes using fungi, but the capacity of such microorganisms to modify the structure of this class of natural products is even greater.

Other modifications reported include ring rearrangements with formation of gibberellane [53,56,57,115] and beyerane diterpenes [23,43,116], formation of lactones [21,58,60], epoxidation [60], glycosylation [21], alkene hydrogenation [100], ring opening [53], decarboxylation [60] and the formation of alkenes [43]. Oxidations and ring opening were reported by Fraga et al. When epi-candol A (40) was submitted to biotransformation by the fungus G. fujikuroi, two products were obtained, ent-7 $\beta, 16 \beta, 17$-trihydroxy-kaur-16-ene and fujenoic acid (87) [53]. Fujenoic acid was formed after hydroxylation at C-6, followed by oxidation at C-7 and ring opening (Scheme 12). The fujenoic triacid $\mathbf{8 8}$ is a natural product found in G. fujikuroi and it is an intermediate of gibberellin biosynthesis [117]. To ensure that the product obtained in this biotransformation was not a secondary metabolite of this fungus, the experiment was repeated with isotopic labeling of the substrate [53].

Scheme 12. Ring opening reaction by G. fujikuroi.

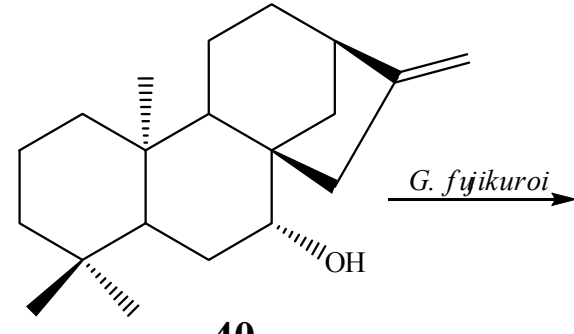

40

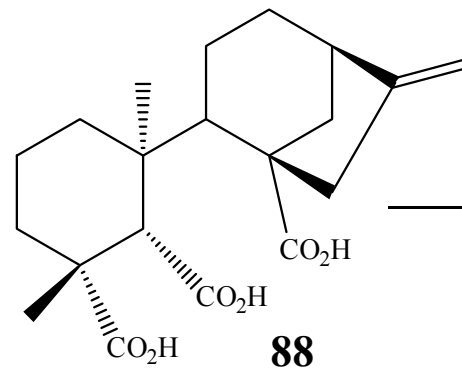

88

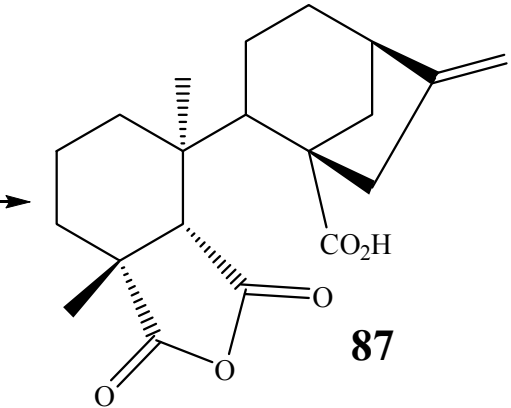

Another interesting modification reported is the decarboxylation of ent-15ß-hydroxy-kaur-9(11),16dien-19-oic acid (43) with the fungus $F$. fujikuroi. Products bearing new hydroxylations at C3 $\beta$ and C$6 \beta$ and 19,6 $\alpha$-lactone derivatives were obtained, besides an oxidative decarboxylation product affording a compound $\mathbf{8 9}$ bearing a 4,18-double bond (Scheme 13). A mechanism for the formation of this exocyclic double bond after decarboxylation of the carboxyl group has been proposed by Fraga et al. [60].

Scheme 13. Formation of exocyclic double bond at C-4 by F. fujikuroi.

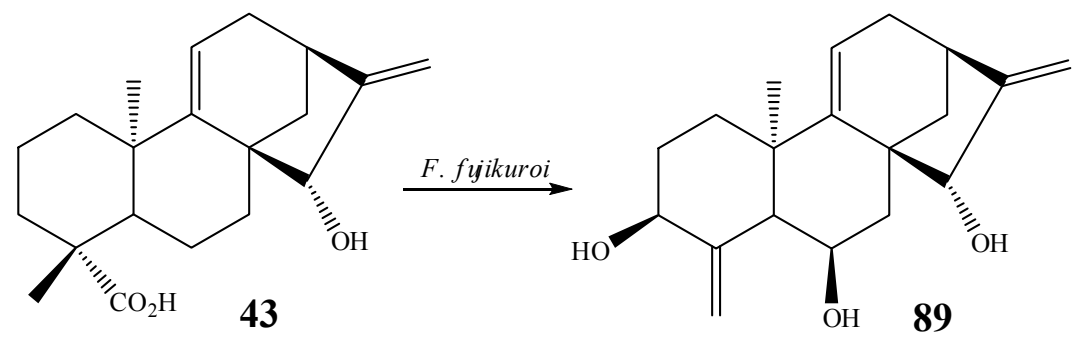


Isomerization of the exocyclic double bond in $\mathbf{9 0}$ of the starting material epicandicandiol (91) has been reported utilizing the fungus $M$. plumbeus [102]. The formation of a double bond was also observed in the biotransformation by the fungus $R$. stolonifer in another position of the kaurane skeleton (between carbons 9 and 11, i.e., compounds 1, 92, Scheme 14) [104].

Scheme 14. Double bond formation by M. plumbeus and R. stolonifer.
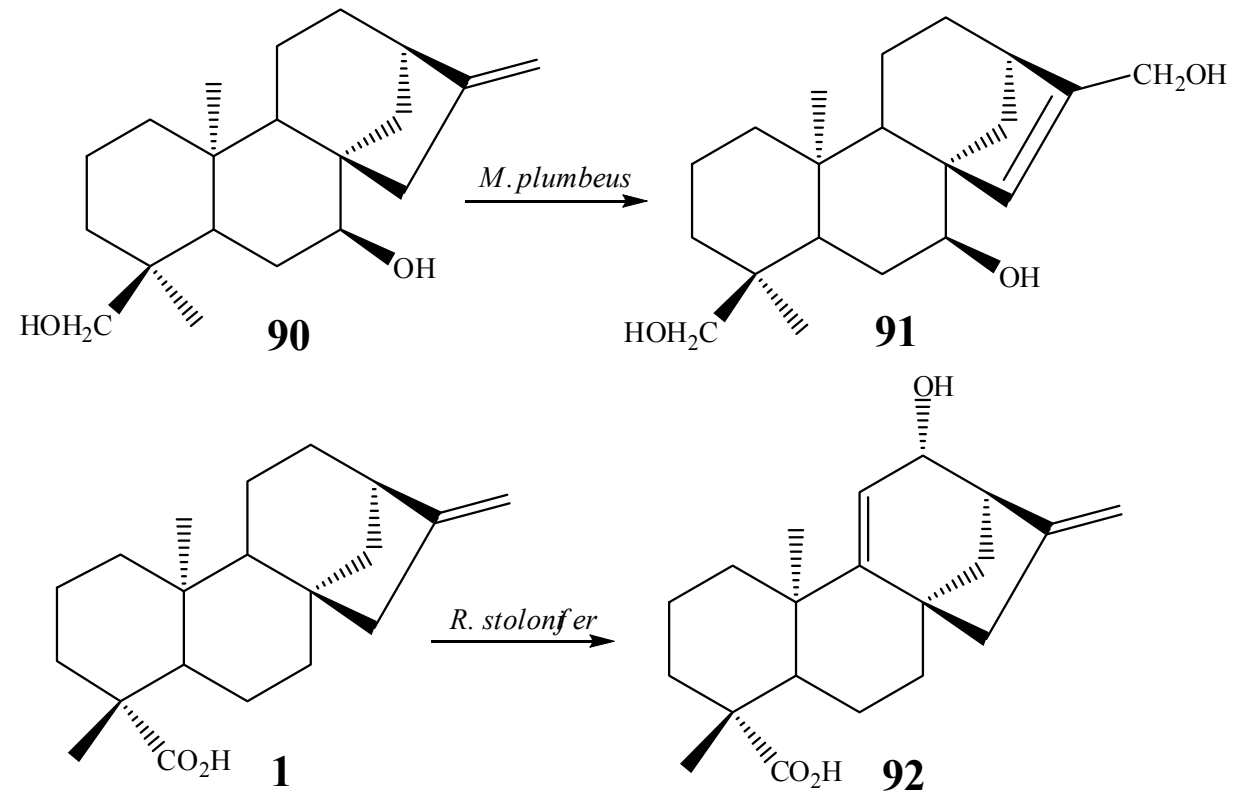

In addition to the already discussed changes that can occur in the kaurane skeleton, rearrangements leading to other classes of diterpenes have been reported, being interesting as a methodology to synthesize compounds with other skeletons. For instance, the diterpene ent-15-oxo-kaur-16-ene furnished seven biotransformation products, five of them (93-97, Figure 18) belonging to the gibberellin group by the action of the fungus G. fujikuroi [57].

Figure 18. Rearrangement product formed in the biotransformation of 7-oxo-ent-kaur-16ene by G. fujikuroi.

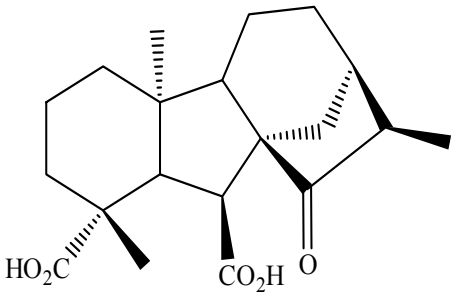

93

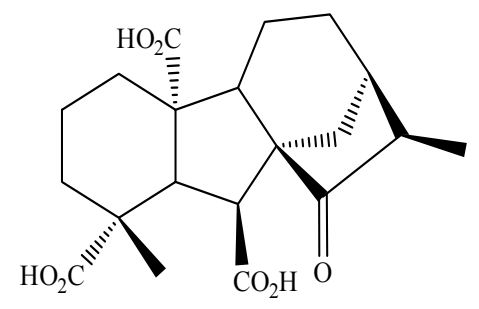

94

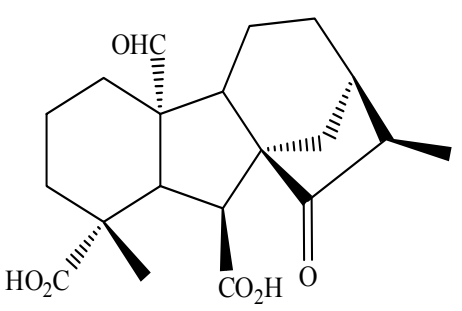

95

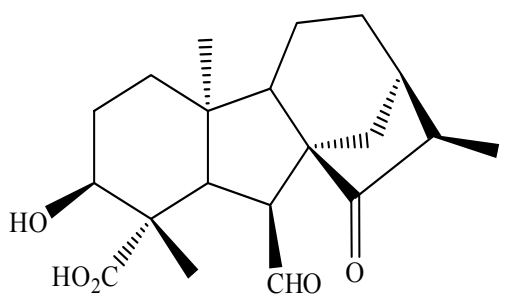

96

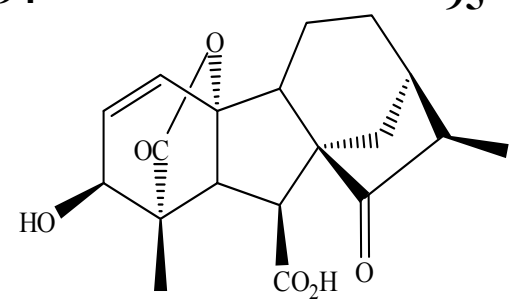

97 
Another interesting rearrangement of kaurane diterpenes can be exemplified by the biotransformation of steviol-16 $\alpha, 17$-epoxide by $C$. bainieri that furnished ent-9 $\alpha, 17$-dihydroxy-16ketobeyeran-19-oic acid (98), ent-1 $\beta, 17$-dihydroxy-16-ketobeyeran-19-oic acid (99), ent-7 $\alpha, 17$ dihydroxy-16-ketobeyeran-19-oic acid (100) and ent-7ß,17-dihydroxy-16-ketobeyeran-19-oic acid (101) as rearrangement products displying beyerane skeletons (Figure 19) [43].

Figure 19. Chemical structures of rearrangements products obtained from biotransformation by C. bainieri.

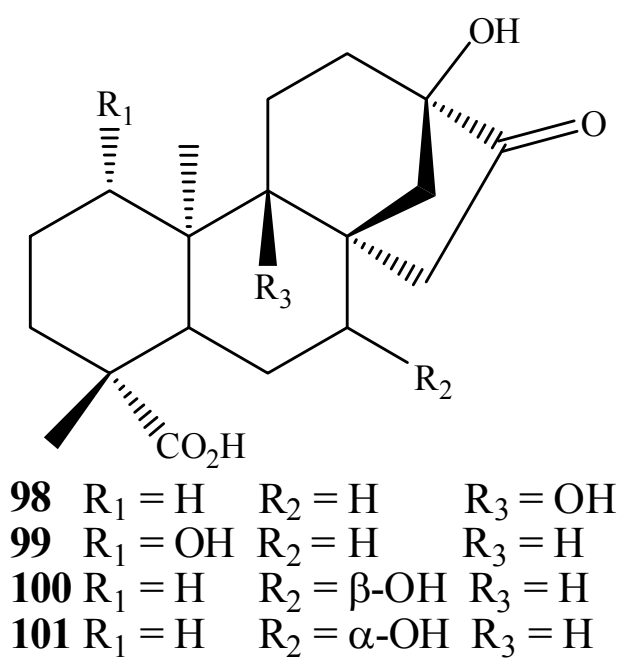

\section{Final Remarks}

In this review we have showed selected examples of the structural diversity of kaurane diterpenes obtained as a result of biotransformations by filamentous fungi species. Kaurane skeletons have been functionalized on all four rings and in both, $\alpha$ - and $\beta$-stereochemistry at most of the carbons. Such functionalizations of molecules represent an important strategy in the discovery of new drugs with different activities.

Advances in the field of natural products research already allow working with intelligent design of novel pharmacologically active molecules. Pharmaceutical chemistry is one of the fields that deal with the creation of molecules for specific goals. The success of this approach is highly dependent on a complete understanding of substrate skeletal susceptibility to chemical and microbial funcionalizations. The intersection of information about structural features responsible for biological activities and fungal species able to activate the structures in these required positions can be extremely useful for the intelligent design of new kaurane derivatives with enhanced biological activities.

Biotransformation of terpenes is an area that is expected to grow since it remains as a modern alternative in the gap left by organic synthesis, due to the possibility of activating methylene carbons. The scope of biotransformations and the green features associated to this methodology are also expected to be a lever feature for increasing the scope of biotransformations used in the new drug discovery process. 


\section{Acknowledgments}

To FAPEMIG, for grants and precious financial help (Project CEX - PPM-00392-13). To CAPES and $\mathrm{CNPq}$ for grants and fellowships.

\section{Conflicts of Interest}

The authors declare no conflict of interest.

\section{References}

1. Leresche, J.E.; Meyer, H.-P. Chemocatalysis and Biocatalysis (Biotransformation): Some thoughts of a chemist and of a biotechnologist. Org. Process Res. Dev. 2006, 10, 572-580.

2. Serra, S.; Fuganti, C.; Brenna, E. Biocatalytic preparation of natural flavors and fragrances. Trends Biotechnol. 2005, 23, 193-198.

3. Schwab, W.; Fuchs, C.; Huang, F.C.; Transformation of terpenes into fine chemicals. Eur. J. Lipid Sci. Technol. 2013, 115, 3-8.

4. Prieto, S.; Gloria, A.; Perea, V.; Janeth, A.; Ortiz, L.; Claudia, C. Microbial biotransformation of (R)-(+)-limonene by Penicillium digitatum DSM 62840 for producing (R)-(+)-terpineol. Vitae 2011, 18, 136-172.

5. Barrero, A.F.; Oltra, J.E.; Raslan, D.S.; Saúde, D.A. Microbial transformation of sesquiterpene lactones by the fungi Cunninghamella echinulata and Rhizopus oryzae. J. Nat. Prod. 1999, 62, 726-729.

6. Rocha, B.A.; Pupo, M.T.; Antonucci, G.A.; Sampaio, S.V.; Paiva, R.D.M.A.; Said, S.; da Costa, F.B. Microbial transformation of the sesquiterpene lactone tagitinin $\mathrm{C}$ by the fungus Aspergillus terreus. J. Ind. Microbiol. Biotechnol. 2012, 39, 1719-1724.

7. Martins, L.R.; Takahashi, J.A. Rearrangement and oxidation of $\beta$-amyrin promoted by growing cells of Lecanicillium muscarinium. Nat. Prod. Res. 2010, 24, 767-774.

8. Schmeda-Hirschmann, G.; Aranda, C.; Kurina, M.; Rodríguez, J.A.; Theoduloz, C. Biotransformations of imbricatolic acid by Aspergillus niger and Rhizopus nigricans cultures. Molecules 2007, 12, 1092-1100.

9. Fraga, B.M.; Gonzalez-Vallejo, V.; Guillermo, R. On the biotransformation of ent-trachylobane to ent-kaur-11-ene diterpenes. J. Nat. Prod. 2011, 74, 1985-1989.

10. Fraga, B.M.; Bressa, C.; González-Vallejo, V.; González, P.; Guillermo, R. Biotransformation of ent-kaur-16-ene and ent-trachylobane $7 \beta$-acetoxy derivatives by the fungus Gibberella fujikuroi (Fusarium fujikuroi). Phytochemistry 2012, 81, 60-70.

11. Hanson, J.R.; Reese, P.B.; Takahashi, J.A.; Wilson, M.R. Biotransformation of some stemodane diterpenoids by Cephalosporium aphidicola. Phytochemistry 1994, 36, 1391-1393.

12. Hanson, J.R.; Jarvis, A.G.; Laboret, F.; Takahashi, J. The incubation of 3a,16ß-dihydroxyaphidicolane with Cephalosporium aphidicola. Phytochemistry 1995, 38, 73-75.

13. Fraga, B.M.; González, P.; Hernández, M.G.; Chamy, M.C.; Garbarino, J.A. Microbiological transformation of an ent-pimaradiene hydrocarbon by Gibberella fujikuroi. Phytochem. Lett. 2009, 2, 201-203. 
14. Fraga, B.M.; Hernández, M.G.; Artega, J.M.; Suárez, S. The microbiological transformation of the diterpenes dehydroabietanol and teideadiol by Mucor plumbeus. Phytochemistry 2003, 63, $663-668$.

15. Fraga, B.M.; Gonzalez, P.; Gonzalez-Vallejo, V.; Guillermo, R.; Diaz, L.N. Biotransformation of 7 $\alpha$-hydroxy- and 7-oxo-ent-atis-16-ene derivatives by the fungus Gibberella fujikuroi. Phytochemistry 2010, 71, 1313-1321.

16. Oliveira, B.H.; Strapasson, R.A. Biotransformation of isosteviol by Fusarium verticilloides. Phytochemistry 1996, 43, 393-395.

17. Hu, S.; Tian, X.; Zhu, W.; Fang, Q. Biotransformation of $2 \alpha, 5 \alpha, 10 \beta, 14 \beta$-Tetraacetoxy-4(20),11taxadiene by the Fungi Cunninghamella elegans and Cunninghamella echinulata. J. Nat. Prod. 1996, 59, 1003-1009.

18. Ghisalberti, E.L. The biological activity of natural occurring kaurane diterpenes. Fitoterapia 1997, 68, 303-325.

19. Chavan, M.J.; Wakte, P.S.; Shinde, D.B. Analgesic and anti-inflammatory activities of 18-acetoxy-ent-kaur-16-ene from Annona squamosa L. bark. Inflammopharmacol. 2011, 19, $111-115$.

20. Ohkoshi, E.; Kamo, S.; Makino, M.; Fujimoto, Y. ent-Kaurenoic acids from Mikania hirsutissima (Compositae). Phytochemistry 2004, 65, 885-890.

21. Fraga, B.M.; Alfonso, I.; Gonzalez-Vallejo, V.; Guillermo, R. Microbial transformation of two 15a-hydroxy-ent-kaur-16-ene diterpenes by Mucor plumbeus. Tetrahedron 2010, 66, 227-234.

22. Ko, H.-H.; Chang, W.-L.; Lu, T.-M. Antityrosinase and antioxidant effects of ent-kaurane diterpenes from leaves of Broussonetia papyrifera. J. Nat. Prod. 2008, 71, 1930-1933.

23. Yang, L.-M.; Hsu, F.-L.; Chang, S.-F.; Cheng, J.-T.; Hsu, C.-Y.; Liu, P.-C.; Lin, S.-J. Microbial metabolism of steviol and steviol-16a,17-epoxide. Phytochemistry 2007, 68, 562-570.

24. Cheenpracha, S.; Yodsaoue, O.; Karalai, C.; Ponglimanont, C.; Subhadhirasakul, S.; TewtrakuL, S.; Kanjana-opas, A. Potential anti-allergic ent-kaurene diterpenes from the bark of Suregada multiflora. Phytochemistry 2006, 67, 2630-2634.

25. Kim, S.; Na, M.; Oh, H.; Jang, J.; Sohn, C.B.; Kim, B.Y.; Oh, W.K.; Ahn, J.S. PTP1B inhibitory activity of kaurane diterpenes isolated from Siegesbeckia glabrescens. J. Enzyme Inhib. Med. Chem. 2006, 21, 379-383.

26. Rocha, A.D.; dos Santos, G.C.; Fernandes, N.G.; Pfenning, L.H.; Takahashi, J.A.; Boaventura, M.A.D. Hydroxylation at carbon-2 of ent-16-oxo-17-norkauran-19-oic acid by Fusarium proliferatum. J. Nat. Prod. 2010, 73, 1431-1433.

27. Marquina, S.; Parra, J.L.; González, M.; Zamilpa, A.; Escalante, J.; Trejo-Hernández, M.R.; Álvarez, L. Hydroxylation of the diterpenes ent-kaur-16-en-19-oic and ent-beyer-15-en-19-oic acids by the fungus Aspergillus niger. Phytochemistry 2009, 70, 2017-2022.

28. Rocha, D.; Takahashi, J.A.; Boaventura, M.A.D. Di- and tri-hydroxylated kaurane derivatives from microbial transformation of ent-kaur-16-en-19-ol by Cephalosporium aphidicola and their allelopathic activity on Lactuca sativa (Lettuce). Eclet. Quim. 2009, 34, 57-62.

29. Ambrosio, S.R.; Furtado, N.A.; de Oliveira, D.C.; da Costa, F.B.; Martins, C.H.; de Carvalho, T.C.; Porto, T.S.; Veneziani, R.C. Antimicrobial activity of kaurane diterpenes against oral pathogens. Z. Naturforsch. 2008, 63, 326-330. 
30. Alves, T.M.A.; Chaves, P.P.G.; Santos, L.M.S.T.; Nagem, T.J.; Murta, S.M.F.; Ceravolo, I.P.; Romanha, A.J.; Zani, C.L. A diterpene from Mikania obtusata active on Trypanosoma cruzi. Planta Med. 1995, 61, 85-87.

31. Vieira, H.S.; Takahashi, J.A.; Boaventura, M.A.D. Novel derivatives of ent-17,19-dihydroxy$16 \beta \mathrm{H}-\mathrm{kaurane}$ obtained by biotransformation with Verticillium lecanii. J. Agric. Food Chem. 2002, 50, 3704-3707.

32. Slimestad, R.; Marston, A.; Mavi, S.; Hostettmann, K. Larvicidal constituents of Melantheria albinervia. Planta Med. 1995, 61, 562-563.

33. Padmaja, V.; Thankamany, V.; Hara, N.; Fujimoto, Y.; Hisham, A. Biological activities of Annona glabra. J. Ethnopharmacol. 1995, 48, 21-24.

34. Block, L.C.; Santos, A.R.S.; de Souza, M.M.; Scheidt, C.; Yunes, R.A.; Santos, M.A.; Monache, F.D.; Cechinel-Filho, V. Chemical and pharmacological examination of antinociceptive constituents of Wedelia paludosa. J. Ethnopharmacol. 1998, 61, 85-89.

35. Costa-Lotufo, L.V.; Cunha, G.M.A.; Farias, P.A.M.; Viana, G.S.B.; Pessoa, C.; Moraes, M.O.; Silveira, E.R.; Gramosa, N.V.; Rao, V.S.N. The cytotoxic and embryotoxic effects of kaurenoic acid, a diterpene isolated from Copaifera langsdorffii oleo-resin. Toxicon 2002, 40, 1231-1234.

36. Cavalcanti, B.C.; Costa-lotufo, L.V.; Moraes, M.O.; Burbano, R.R.; Silveira, E.R.; Cunha, K.M.A.; Rao, V.S.N.; Moura, D.J.; Rosa, R.M.; Henriques, J.A.P.; et al. Genotoxicity evaluation of kaurenoic acid, a bioactive diterpenoid present in copaiba oil. Food Chem. Toxicol. 2006, 44, 388-392.

37. De Carli, R.B.G.; Siqueira, P.R.A.; Kaiser, M.L.; Freitas, R.A.; de Souza, M.M.; Cechinel-Filho, V.; Lucinda-Silva, R.M. Topical anti-inflammatory effect of creams containing kaurenoic acid isolated from Wedelia paludosa in Mice. Lat. Am. J. Pharm. 2009, 28, 594-598.

38. Guillopé, R.; Escobar-Khondiker, M.; Guérineau, V.; Laprévote, O.; Höglinger, G.U.; Champy, P. Kaurenoic acid from pulp of Annona cherimolia in regard to Annonaceae-induced Parkinsonism. Phytother. Res. 2011, 25, 1861-1864.

39. Chang, F.-R.; Yang, P.-Y.; Lin, J.-Y.; Lee, K.-H.; Wu, Y.-C. Bioactive kaurane diterpenoids from Annona glabra. J. Nat. Prod. 1998, 61, 437-439.

40. Paiva, L.A.; Gurgel, L.A.; Silva, R.M.; Tome, A.R.; Gramosa, N.V.; Silveira, E.R.; Santos, F.A.; RAO, V.S. Anti-inflammatory effect of kaurenoic acid, a diterpene from Copaifera langsdorffii on acetic acid-induced colitis in rats. Vascul. Pharmacol. 2002, 39, 303-307.

41. Takahashi, J.A.; Vieira, H.S.; Silva, E.A.; Boaventura, M.A.D.; Oliveira, A.B.; Chiari, E. Preparation and activity of diterpenoids against trypomastigotes of Trypanosoma cruzi. Rev. Bras. Farmacogn. 2002, 12(supl. 1), 118-120.

42. Zhang, Y.-H.; Peng, H.-Y.; Xia, G.-H.; Wang, M.-Y.; Han, Y. Anticancer effect of two diterpenoid compounds isolated from Annona glabra Linn. Acta Pharmacol. Sin. 2004, 25, 937-942.

43. Chang, S.-F.; Yang, L.-M.; Hsu, F.-L.; Hsu, J.-Y.; Liaw, J.-H.; Lin, S.-J. Transformation of steviol-16 $\alpha, 17$-epoxide by Streptomyces griseus and Cunninghamella bainieri. J. Nat. Prod. 2006, 69, 1450-1455.

44. Sun, H.-D.; Huang, S.-X.; Han, Q.-B. Diterpenoids from Isodon species and their biological activities. Nat. Prod. Rep. 2006, 23, 673-698. 
45. Urzúa, A.; Rezende, M.C.; Mascayano, C.; Vásquez, L. A Structure-activity study of antibacterial diterpenoids. Molecules 2008, 13, 882-891.

46. Fujita, E.; Nagao,Y.; Kaneko,K.; Nakazawa, S.; Kuroda, H. The antitumor and antibacterial activity of the Isodon diterpenoids. Chem. Pharm. Bull. 1976, 24, 2118-2127.

47. Lin, L.; Gao, Q.; Cui, C.; Zhao, H.; Fu, L.; Chen, L.; Yang, B.; Luo, W.; Zhao, M. Isolation and identification of ent-kaurane-type diterpenoids from Rabdosia serra (MAXIM.) HARA leaf and their inhibitory activities against HepG-2, MCF-7, and HL-60 cell lines. Food Chem. 2012, 131, 1009-1014.

48. Nagao, Y.; Ito, N.; Kohno, T.; Kuroda, H.; Fujita, E. Antitumor activity of Rabdosia and Teucrium diterpenoids against P 388 lymphocytic leukemia in mice. Chem. Pharm. Bull. 1982, 30, 727-729.

49. Dutta, T.K.; Samanta, T.B. Bioconversion of progesterone by the activated immobilized conidia of Aspergillus ochraceus. Curr. Microbiol. 1999, 39, 309-312.

50. Lisurek, M; Bernhardt, R. Modulation of aldosterone and cortisol synthesis on the molecular level. Mol. Cell. Endocrinol. 2004, 215, 149-159.

51. Kvas, M.; Marasas, W.F.O.; Wingfield, B.D.; Wingfield, M.J.; Steenkamp, E.T. Diversity and evolution of Fusarium species in the Gibberella fujikuroi complex. Fungal Divers. 2009, 34, $1-21$.

52. Ávalos, J.; Cerdá-Olmedo, E.; Reyes, F.; Barrero, A.F. Gibberellins and other metabolites of Fusarium fujikuroi and related fungi. Curr. Org. Chem. 2007, 11, 721-737.

53. Fraga, B.M.; Bressa, C.; González, P.; Guillermo, R.; Hernández, M.G.; Suárez, S. The microbiological transformation of $7 \alpha$-hydroxy-ent-kaur-16-ene derivatives by Gibberella fujikuroi. Phytochemistry 2007, 68, 1557-1563.

54. Hedden, P. The oxidases of gibberellin biosynthesis: Their function and mechanism. Physiol. Plant. 1997, 101, 709-719.

55. Alam, M.; Hanson, J.R.; Sarah, F. The biotransformation of some 6-substituted ent-kaur-16-enes by Gibberella fujikuroi. Phytochemistry 1991, 30, 807-809.

56. Fraga, B.M.; Gonzalez, P.; Guillermo, R.; Hanson, J.R.; Hernandez, M.G.; Takahashi, J.A. The microbiological transformation of two ent-16ß,17-epoxykaurane derivatives by Gibberella fujikuroi. Phytochemistry 1994, 37, 717-721.

57. Fraga, B.M.; Gonzalez, P.; Guillermo, R.; Hernandez, M.G.; Perales, A. The chemical and microbiological preparation of 15-oxo-gibberellin derivatives. Tetrahedron 1995, 51, 10053-10064.

58. Fraga, B.M.; Gonzalez, P.; Hernandez, M.G.J.; Suárez, S. Biotransformation of 7-oxo-ent-kaur16-ene derivatives by Gibberella fujikuroi. Tetrahedron 2005, 61, 5623-5632.

59. García-Granado, A.; Martinez, A.; Onorato, M.E.; Ruiz, M.L.; Sánches, J.M.; Arias, J.M. Biotransformation of highly substituted ent-kaur-16-enes by Rhizopus nigricans. Phytochemistry 1990, 29, 121-126.

60. Fraga, B.M.; González-Vallejo, V.; Guillermo, R.; Amaro-Luis, J.M. Microbiological transformation of two 15a-hydroxy-ent-kaur-9(11),16-diene derivatives by the fungus Fusarium fujikuroi. Phytochemistry 2013, 89, 39-46.

61. Fraga, B.M.; Hernández, M.G.; Guillermo, R. The biotransformation of two 3,15-oxygenate ent-kaurane derivatives by Gibberella fujikuroi. J. Nat. Prod. 1996, 59, 952-957. 
62. García-Granado, A.; Martinez, A.; Ortiz, A.; Onorato, M.E.; Arias, J.M. Microbiological transformation of tetracyclic diterpenes: Conversion of ent-kaurenones by Curvularia and Rhizopus strains. J. Nat. Prod. 1990, 53, 441-450.

63. Giles, P.M. Revised section F: Natural products and related compounds. Pure Appl. Chem. 1999, 71, 587-643.

64. Taveepanich, S.; Muangsin, N.; Saithong, S.; Pakawatchai, C.; Chaichit, N.; Roengsumran, S.; Petsom, A. Biotransformation of ent-kaur-16-en-19-oic acid by Absidia blakesleeana and Rhizopus oligosporus. Nat. Prod. Rep. 2010, 24, 1050-1058.

65. Anderson, A.B.; McCrindle, R.; Turnbull, J.K. Microbiological transformation of 17-norkauran16-one and 16-norphyllocladan-16-one by Aspergillus niger. J. Chem. Soc. Chem. Comm. 1973, 143-144.

66. Beilby, J.P.; Ghisalberti, E.L.; Jefferies, P.R.; Sefton, M.A.; Sheppard, P.N. Microbiological transformation of tetracyclic diterpenes. Tetrahedron Lett. 1973, 14, 2589-2592.

67. Ghisalberti, E.L.; Jefferies, P.R.; Sefton, M.A.; Sheppard, P.N. Microbial transformation of 19-oxigenated ent-kauranes. Tetrahedron 1977, 33, 2451-2456.

68. Boaventura, M.A.D.; Hanson, J.R.; Hitchcock, P.B.; Takahashi, J.A. The biotransformation of ent-19-hydroxykaur-16-en-15-one by Cephalosporium aphidicola. Phytochemistry 1994, 37, 387-389.

69. Oliveira, A.B.; Hanson, J.R.; Takahashi, J.A. The biotransformation of ent-15-oxo-kaur-16-en-19-oic acid and its methyl ester by Cephalosporium aphidicola. Phytochemistry 1995, 40, 439-442.

70. Hanson, J.R.; Hitchcock, P.B.; Takahashi, J.A. Biotransformation of ent-16ß,19dihydroxykaurane by Cephalosporium aphidicola. Phytochemistry 1995, 40, 797-800.

71. El-Emary, N.A.; Kusano, G.; Takemoto, T. Microbial transformation of (-)-kaur-16-en-19-oic acid. Chem. Pharm. Bull. 1976, 24, 1664-1667.

72. Oliveira, B.H.; Filho, J.D.F.; Leal, P.C. Biotransformation os steviol by Aspergillus niger and Fuscarium moniliforme. J. Braz. Chem. Soc. 2005, 16, 210-213.

73. Hanson, J.R.; White, A.F. The transformation of steviol by Gibberella fujikuroi. Tetrahedron 1968, 24, 6291-9293.

74. Hanson, J.R.; Hawker, J.; White, A.F. Studies in terpenoid biosynthesis. Part IX. The sequence of oxidation on ring B in kaurene-gibberellin biosynthesis. J. Chem. Soc. Perkin Trans. 1 1972, 1892-1895.

75. Cook, I.F.; Jefferies, P.R.; Knox, J.R. Acidic ent-kauranoids from the metabolism of ent-kaura2,16-dien-19-ol in Gibberella fujikuroi. Tetrahedron 1975, 31, 251-255.

76. Bearder, J.R.; MacMillan, J.; Wels, C.M. The metabolism of steviol to 13-hydroxylated entgibberellanes and ent-kauranes. Phytochemisty 1975, 14, 1741-1748.

77. Wada, K.; Imai, T.; Shibata, K. Microbial productions of unnatural gibberellins from (-)-kaurene derivatives in Gibberella fujikuroi. Agric. Biol. Chem. 1970, 43, 1157-1158.

78. Wada, K.; Yamashita, H. Synthesis and microbial transformation of ent-12ß-hydroxykaur-16ene. Agric. Biol. Chem. 1980, 44, 2249-2250.

79. Fraga, B.M.; Hanson, J.R.; Hernández, M.G.; Sarah, F.Y. The microbiological transformation of some ent-kaur-16-ene 7-,15- and 18-alcohols by Gibberella fujikuroi. Phytochemistry 1980, 19, 1087-1091. 
80. Fraga, B.M.; González, A.G.; Hanson, J.R.; Hernández, M.G. The microbiological

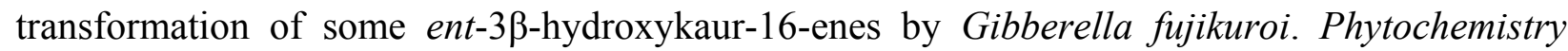
1981, 20, 57-61.

81. Wada, K.; Imai, T.; Yamashita, H. Microbiological production of plant gibberellins and related compounds from ent-kaurane derivatives in Gibberella fujikuroi. Agric. Biol. Chem. 1981, 45, 1833-1842.

82. Fraga, B.M.; González, A.G.; González, P.; Hanson, J.R.; Hernández, M.G.; Hitchcock, P.B. The

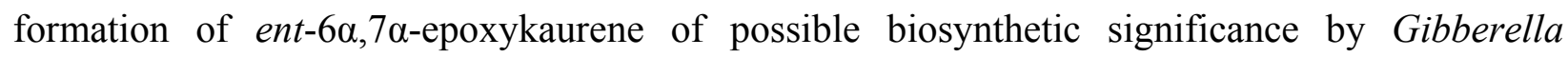
fujikuroi. J. Chem. Soc. Chem. Comm. 1982, 311-312.

83. Beale, M.H.; Bearder, J.R.; Down, G.H.; Hutchison, M.; MacMillan, J.; Phinney, B.O. The biosynthesis of kaurenolide diterpenoids by Gibberella fujikuroi. Phytochemistry 1982, 21, 1279-1287.

84. Shigematsu, Y.; Murofushi, N.; Takahashi, N. Structures of the metabolites from steviol methyl ester by Gibberella fujikuroi. Agric. Biol. Chem. 1982, 46, 2313-2318.

85. Fraga, B.M.; González, A.G.; González, P.; Hanson, J.R.; Hernández, M.G. The microbiological transformation of some ent-kaur-6,16-dienes by Gibberella fujikuroi. Phytochemistry 1983, 22, 691-694.

86. Gaskin, P.; Hutchison, M.; Lewis, N.; MacMillan, J.; Phinney, B.O. Microbiological conversion of 12-oxygenated and other derivatives of ent-kaur-16-en-19-oic acid by Gibberella fujikuori. Phytochemistry 1984, 23, 559-564.

87. Fraga, B.M.; González, P.; Hernández, M.G.; García-Tellado, F.; Perales, A. The microbiological transformation of candidiol, ent-15 $\beta, 18$-dihydroxy-kaur-16-ene, by Gibberella fujikuroi. Phytochemistry 1986, 25, 1235-1237.

88. Fraga, B.M.; Hernández, M.G.; Rodrigues, M.D.; Diaz, C.E.; González, P.; Hanson, J.R. Transformation of ent-kaur-15-ene by Gibberella fujikuroi. Phytochemistry 1987, 26, 1931-1934.

89. Fraga. B.M.; Hernández, M.G.; Diaz, C.E.; González, P.; Guillermo, R. The microbiological transformation of some ent-15ß-hydroxykaurenes by Gibberella fujikuroi. Phytochemistry 1988, 27, 3131-3136.

90. Hanson, J.R.; Oliveira, B.H. The microbiological transformation of steviol derivatives by Rhyzopus nigricans and Gibberella fujikuroi. Phytochemistry 1990, 29, 3805-3807.

91. Fraga, B.M.; Hernández, M.G.; González, P. The microbiological transformation of some ent-7 $\alpha, 15 \beta$-dihydroxykaurene derivatives by Gibberella fujikuroi. Phytochemistry 1991, 30, 2567-2571.

92. Fraga, B.M.; Hernández, M.G.; González, P. Biotransformation of two ent-15 $\beta$-hydroxy-kaur16-ene derivatives by Gibberella fujikuroi. Phytochemistry 1992, 31, 3845-3849.

93. Fraga, B.M.; García-Tellado, F.; González, P.; Hernández, M.G. The chemical and microbiological synthesis of 14-hydroxy-gibberellins. Tetrahedron 1992, 48, 8491-8504.

94. Fraga, B.M.; Hernández, M.G.; García-Tellado, F.; González, P.; Perales, A. The

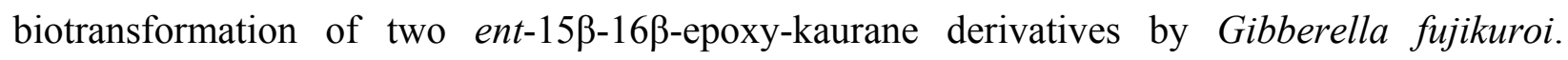
Phytochemistry 1993, 34, 133-138. 
95. Fraga, B.M.; García-Tellado, F.; González, P.; Hernández, M.G. The microbiological transformation of 14ß,19-dihydroxy-ent-kaur-15-ene by Gibberella fujikuroi. Phytochemistry 1993, 34, 1035-1040.

96. Fraga, B.M.; González, P.; Guillermo, R.; Hernández, M.G. A study of microbiological reduction of $\alpha, \beta$-unsaturated carbonyl ent-kaurenes by Gibberella fujikuroi. Tetrahedron 1996, $52,13767-13782$.

97. Fraga, B.M.; González, P.; Guillermo, R.; Hernández, M.G. The formation of an ent-11 $\alpha, 16 \alpha-$ epoxykaurene of biosynthetic significance by Gibberella fujikuroi. Nat. Prod. Lett. 1996, 8, 257-262.

98. Barrero, A.F.; Oltra, J.E.; Cabrera, E.; Reyes, F.; Álvarez, M. Metabolism of gibberellins and ent-kaurenoids in mutants of Gibberella fujikuroi. Phytochemistry 1999, 50, 1133-1140.

99. Barrero, A.F.; Oltra, J.E.; Cerdá-Olmedo, E.; Ávalos, J.; Justicia, J. Microbial transformation of ent-kaurenoic acid and its 15-hydroxy derivatives by the SG138 mutant of Gibberella fujikuroi. J. Nat. Prod. 2001, 64, 222-225.

100. Fraga, B.M.; Guillermo, R.; Hernández, M.G. The microbiological transformation of two 15ß-hydroxy-ent-kaurene diterpenes by Gibberella fujikuroi. J. Nat. Prod. 2004, 67, 64-69.

101. Boaventura, M.A. D.; Oliveira, A.B.; Hanson, J.R.; Hitchcock, P.B.; Takahashi, J.A. The biotransformation of methyl ent-15-oxokaur-16-en-19-oate by Rhizopus stolonifer and Mucor plumbeus. Phytochemistry 1995, 40, 1667-1669.

102. Fraga, B.M.; Álvarez, L.; Suárez, S. Biotransformation of the diterpenes epicandicandiol and candicandiol by Mucor plumbeus. J. Nat. Prod. 2003, 66, 327-331.

103. Pechwang, J.; Sihanonth, P.; Pornpakakul, S.; Muangsin, N.; Piapukiew, J.; Vangnai, A.; Chaichit, N.; Chuchawankul, S.; Petsom, A. Biotransformation of ent-kaur-16-en-19-oic acid by Psilocybe cubensis. Nat. Prod. Rep. 2010, 24, 905-914.

104. Silva, E.A.; Takahashi, J.A.; Boaventura, M.A.D.; Oliveira, A.B. The biotransformation of ent-kaur-16-en-19-oic acid by Rhizopus stolonifer. Phytochemistry 1999, 52, 397-400.

105. Vieira, H.S.; Takahashi, J.A.; Boaventura, M.A.D. Biotransformation of methyl ent-17-hydroxy16ß-kauran-19-oate by Rhizopus stolonifer. Appl. Microbiol. Biotechnol. 2000, 53, 601-604.

106. Hueso-Falcón, I.; Cuadrado, I.; Cidre, F.; Amaro-Luis, J.M.; Ravelo, Á.G.; Estevez-Braun, A.; Heras, B.; Hortelano, S. Synthesis and anti-inflammatory activity of ent-kaurene derivates. Eur. J. Med. Chem. 2011, 46, 1291-1305.

107. Frija, L.M.T.; Frade, R.F.M.; Afonso, C.A.M. Isolation, chemical, and biotransformation routes of labdane-type diterpenes. Chem. Rev. 2011, 111, 4418-4452.

108. Silva, E.O.; Furtado, N.A.J.C.; Aleu, J.; Collado, I.G. Terpenoid biotransformations by Mucor species. Phytochem. Rev. 2013, 12, 857-876.

109. Dewick, P.M. The Mevalonate and Methylerythritol Phosphate Pathaways: Terpenoids and Steroids. In Medicinal Natural Products: A Biosynthetic Approach, 3rd ed.; John Wiley \& Sons Ltd.: Chichester, UK, 2009; pp. 187-310.

110. Sticht, G.; Käferstein, H. Detection of psilocin in body fluids. Forensic. Sci. Int. 2000, 113, 403-407.

111. Kamata, T.; Katagi, M.; Tsuchihashi, H. Metabolism and toxicological analyses of hallucinogenic tryptamine analogues being abused in Japan. Forensic Toxicol. 2010, 28, 1-8. 
112. Carhart-Harris, R.L.; Erritzoea, D.; Williams, T.; Stonea, J.M.; Reeda, L.J.; Colasantia, A.; Tyackea, R.J.; Leechd, R.; Maliziab, A.L.; Murphye, K.; et al. Neural correlates of the psychedelic state as determined by fMRI studies with psilocybin. Proc. Natl. Acad. Sci. USA 2012, 109, 2138-2143.

113. Tanaka, N.; Ooba, N.; Duan, H.; Takaishi, Y.; Nakanishi, Y.; Bastow, K.; Lee, K. Kaurene and abietane diterpenoids from Tripterygium doianum (Celastraceae). Phytochemistry 2004, 65, 2071-2076.

114. Topçu, G.; Ertaş, A.; Öztürk, M.; Dinçel, D.; Kılıç, T.; Halfon, B. Ent-kaurane diterpenoids isolated from Sideritis congesta. Phytochem. Lett. 2011, 4, 436-439.

115. Hanson, J.R. The microbiological transformation of diterpenoids. Nat. Prod. Rep. 1992, 9, 139-151.

116. Milagre, H.M.S.; Martins, L.R.; Takahashi J.A. Novel agents for enzymatic and fungal hydrolysis of stevioside. Braz. J. Microbiol. 2009, 40, 367-372.

117. Rojas, M.C.; Urrutia, O.; Cruz, C.; Gaskin, P.; Tudzynski, B.; Hedden, P. Kaurenolides and fujenoic acids are side products of the gibberellins P450-1 monooxygenase in Gibberella fujikuroi. Phytochemistry 2004, 65, 821-830.

(C) 2014 by the authors; licensee MDPI, Basel, Switzerland. This article is an open access article distributed under the terms and conditions of the Creative Commons Attribution license (http://creativecommons.org/licenses/by/3.0/). 